Review

\title{
Chiral Symmetry Breaking Phenomenon Caused by a Phase Transition
}

\author{
Rui Tamura*, Sekai Iwama and Hiroki Takahashi \\ Graduate School of Human and Environmental Studies, Kyoto University, Kyoto 606-8501, Japan; \\ E-Mails:w.sekai2@gmail.com (S.I.); takahashi@mbox.kudpc.kyoto-u.ac.jp (H.T.) \\ * Author to whom correspondence should be addressed; E-Mail: tamura-r@ mbox.kudpc.kyoto-u.ac.jp.
}

Received: 30 December 2009; in revised form: 9 February 2010 / Accepted: 15 February 2010 /

Published: 17 February 2010

\begin{abstract}
We report the mechanism and scope of "preferential enrichment", which is an unusual symmetry-breaking enantiomeric resolution phenomenon that is initiated by the solvent-assisted solid-to-solid transformation of a metastable polymorphic form into a thermodynamically stable one during crystallization from the supersaturated solution of certain kinds of racemic mixed crystals (i.e., solid solutions or pseudoracemates) composed of two enantiomers. The mechanism can well be interpreted in terms of a symmetrybreaking complexity phenomenon involving multistage processes that affect each other.
\end{abstract}

Keywords: preferential enrichment; enantiomeric resolution; optical resolution; chiral separation; polymorphic transition; epitaxial transition; mixed crystal

\section{Introduction}

Nowadays, a concept of nonlinear "complexity" theory has been recognized to govern various dynamic behaviors in both natural and social sciences. In the complexity system, symmetry is easily broken by the phase transition between two chaotic or metastable states [1-3]. Therefore, it might be possible to observe the symmetry-breaking as a chemical complexity phenomenon by using appropriate probes such as chirality (Figure 1).

Crystallization can be regarded as an event of complexity in terms of the kinetic behavior, far from thermal equilibrium, involving formation of metastable prenucleation molecular aggregates, nucleation and crystal growth, and polymorphic transition. Furthermore, these processes affect each other in 
feedback and feedforward manners. Accordingly, crystallization is strongly affected by the surrounding conditions such as additives (seed crystals and impurities), solvent, temperature, concentration, and so on.

Figure 1. Concept for observing a symmetry-breaking phenomenon induced by phase transition between two metastable phases, leading to the finding of an unusual enantiomeric resolution phenomenon "preferential enrichment".

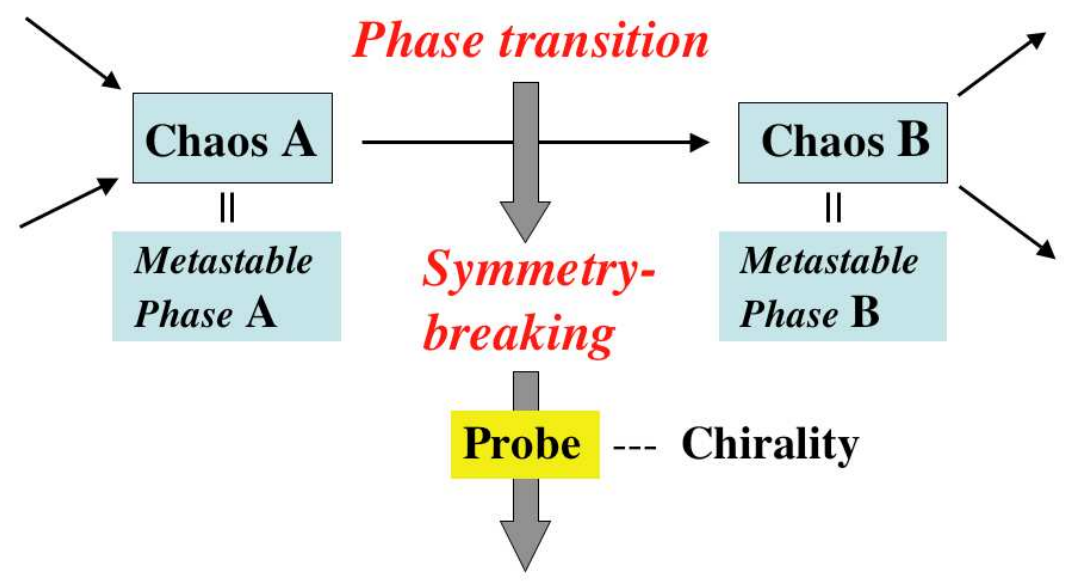

\section{Enantiomeric Resolution Phenomenon "Preferential Enrichment"}

In this context, crystallization of the racemates of chiral compounds is expected to afford a chance of observing a symmetry-breaking in chirality [4]. However, until our discovery of "preferential enrichment", an unusually symmetry-breaking enantiomeric resolution phenomenon observed upon recrystallization of organic racemic crystals [5-8], this fascinating subject was not realized.

The methods for enantiomeric resolution of racemates comprising nonracemizable enantiomers by crystallization are classified into two categories [9]; one is an indirect method using an external chiral element, such as a diastereomeric salt formation followed by fractional crystallization [10-13] or a diastereoselective host-guest inclusion complexation [14], and the other is a straightforward method to separate enantiomers by crystallization in the absence of an external chiral element. As the typical example of this latter category, well known is the "preferential crystallization" method to resolve $a$ racemic conglomerate composed of a mixture of homochiral $R$ and $S$ crystals, in which by repeating crystallization from the supersaturated solution with the aid of its enantiopure seed crystals the enantiomerically enriched crystals are efficiently deposited in the alternating chirality sense [15-18]. However, the racemates existing as a racemic conglomerate occupy only less than $10 \%$ of the characterized crystalline racemates, and more than $90 \%$ of them are supposed to belong to racemic crystals [19], which are further classified into either a racemic compound consisting of a regular packing of a pair of $R$ and $S$ enantiomers or a racemic mixed crystal (in other words, a pseudoracemate or a solid solution) composed of a random alignment of the two enantiomers in the defined positions (Figure 2) [9]. It has been believed over a century that there is no way for resolution of these racemic crystals by simple crystallization in the absence of an external chiral element. Accordingly, if one 
could find out a spontaneous enantiomeric resolution phenomenon for these racemic crystals, it would provide a great impact on industrial as well as academic communities.

Figure 2. General packing modes and binary melting-point phase diagrams of two enantiomers in (a) a racemic conglomerate, (b) a racemic compound, and (c) a racemic mixed crystal. The racemic mixed crystal is further classified into i) disordered type, ii) fairly ordered one (a racemic conglomerate type), iii) fairly ordered one (a racemic compound type), or iv) highly ordered one.

(a)

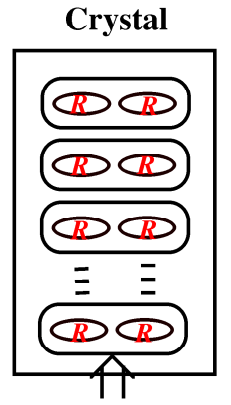

Unit cell (b)

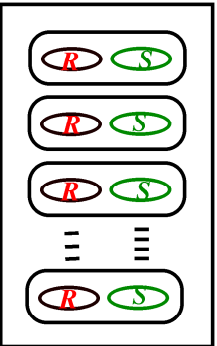

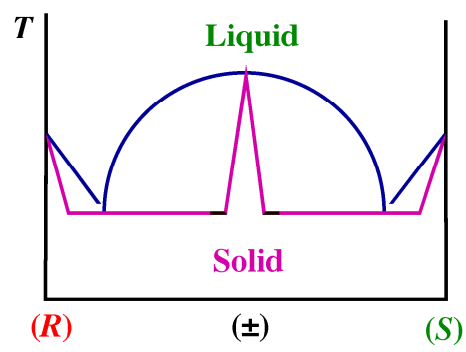

(c)

i)

ii)
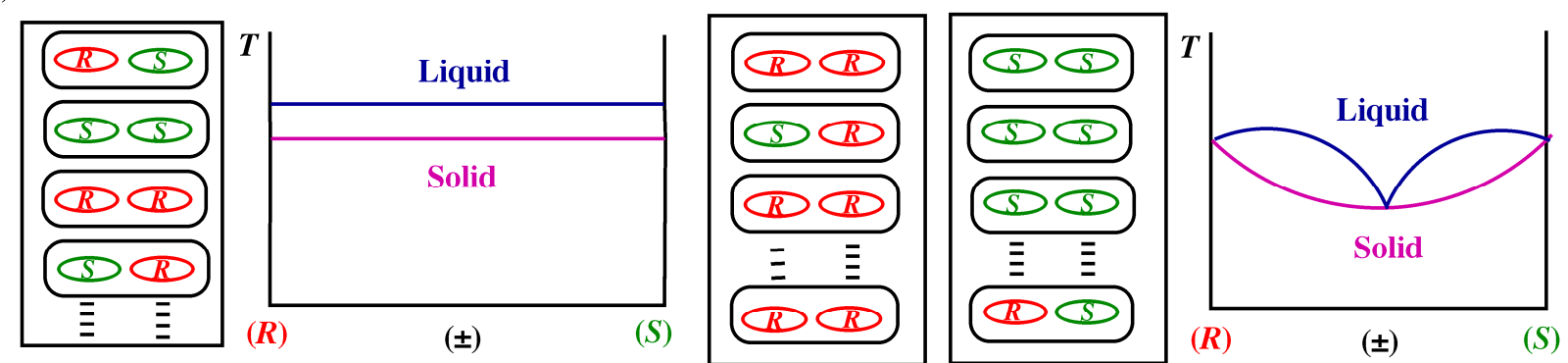

iii)
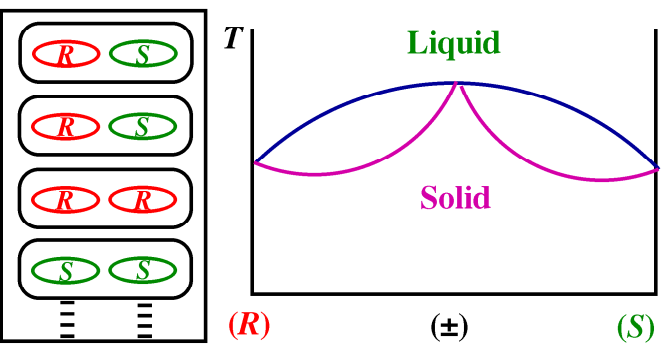

iv)
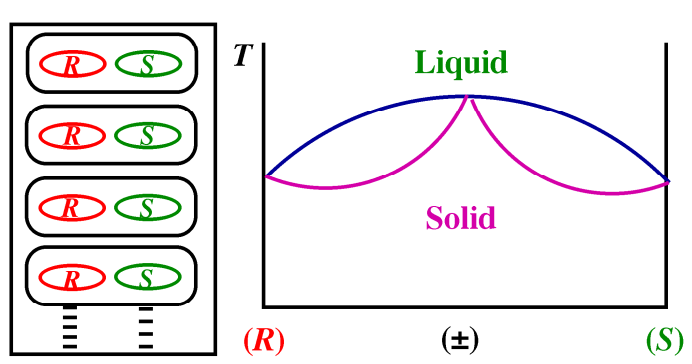

In 1996 we found the first instance where such an ideal enantiomeric resolution by simple recrystallization of a series of racemic crystals is feasible [5,6]. In 1998, this new phenomenon was referred to as preferential enrichment [20], which is completely opposed to preferential crystallization; in preferential enrichment it is in the mother liquor that substantial enantiomeric enrichment occurs by recrystallization, and at the same time slight enrichment of the opposite enantiomer always occurs in the deposited crystals (Figure 3). In 2002, we proposed the mechanism of preferential enrichment in terms of a unique polymorphic transition during crystallization [21,22]. Later, on the basis of the proposed mechanism of this polymorphic transition, we have successfully induced and inhibited the occurrence of preferential enrichment by controlling the mode of the polymorphic transition with 
appropriate seed crystals [23,24]. We have also observed the flexibility in the mode of polymorphic transition that can induce preferential enrichment [24,25]. These results strongly support our proposed mechanism of preferential enrichment.

Figure 3. Features of preferential enrichment.

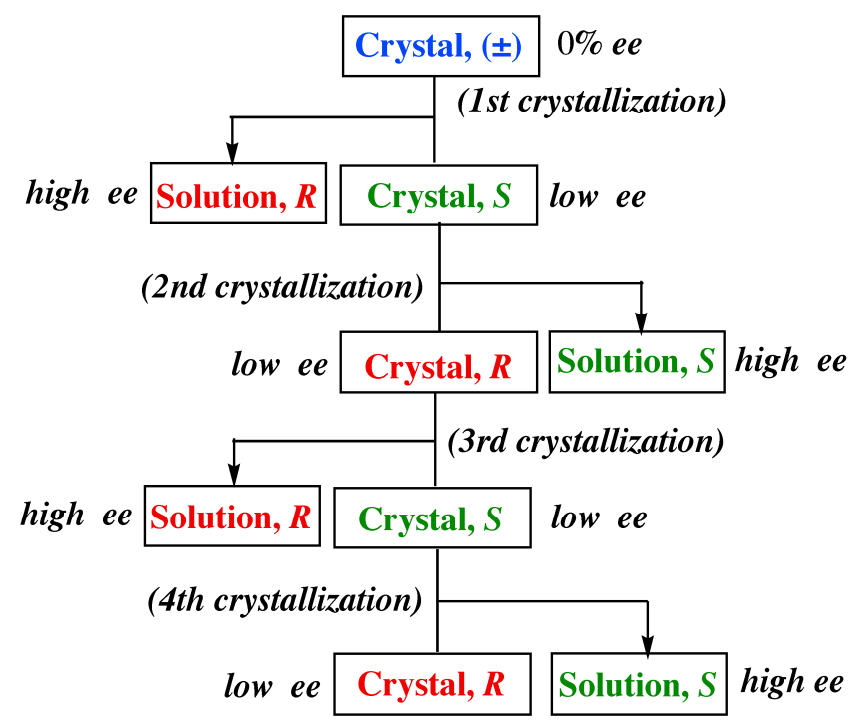

After a thorough investigation on the mechanism, preferential enrichment has been found to be initiated by the solvent-assisted solid-to-solid transformation of a metastable polymorphic form into a thermodynamically stable one during crystallization from the supersaturated solution of certain kinds of racemic mixed crystals (i.e., solid solutions or pseudoracemates) composed of two enantiomers without the aid of any external chiral element [5-8]. Most notably, this polymorphic transition process is followed by partial crystal disintegration inside the transformed crystal lattice to release the excess enantiomer into solution until the deposited crystals are slightly enriched with the opposite enantiomer ( $<10 \% e e)$, with full reproducibility [5-8]. Thus, the interplay of i) such a solvent-assisted solid-tosolid polymorphic transition, ii) the subsequent selective redissolution process of the excess enantiomer, which arises from the increasing solubility of the sample with increasing ee value, and iii) the resulting deposition of mixed crystals, which can retain the resulting fairly-random alignment of two enantiomers inside the crystal lattice to memorize an event of chiral symmetry-breaking, has turned out to be responsible for this unique enantiomeric resolution phenomenon [5-8].

Consequently, we have recognized that preferential enrichment is ascribed to an event of complexity that allows the chiral symmetry-breaking by a phase transition. Namely, dissipative selforganization, emergence of new order and chaos, phase transition between chaos and/or order, and increasing returns in complexity correspond to molecular aggregation, formation of metastable phases, polymorphic transition, and substantial enrichment of one enantiomer in preferential enrichment, respectively [1-3].

In this article, we describe the features, mechanism, requirements, and scope of preferential enrichment. 


\section{Features of Preferential Enrichment}

Figure 4 exhibits a typical example of preferential enrichment for nearly racemic $\mathrm{NNMe}_{3}$ (Figure 5) which is a linear asymmetric secondary alcohol containing a glycerol moiety, an amide group, and a trimethylammonium $p$-nitrobenzenesulfonate structure [21]. This compound was synthesized from the racemic epichlorohydrin. But the crystalline sample obtained after the final synthetic procedure was no more racemic but contained either enantiomer in small excess. Namely preferential enrichment already occurred during the final synthetic procedure. By recrystallization of thus obtained $S$-rich crystals of $2.5 \%$ ee from ethanol under 20 -fold supersaturated conditions, substantial enrichment of the same $S$ enantiomer occurs in the mother liquor. At the same time, slight enrichment of the opposite $R$ enantiomer occurs in the deposited crystals. Accordingly, by repeating recrystallization and filtration, alternating enrichment of two enantiomers occurs largely in the mother liquors and slightly in the deposited crystals. These are the most important and fully reproducible features of preferential enrichment. Thus, by collecting the enantiomerically enriched mother liquors with the same handedness, very efficient separation of the two enantiomers ( $>96 \%$ ee) has been easily achieved.

Figure 4. Preferential enrichment of $\mathrm{NNMe}_{3}$. Conditions: ${ }^{a} \mathrm{EtOH}(32 \mathrm{~mL})$ at $25{ }^{\circ} \mathrm{C}$ for 4 days; ${ }^{\boldsymbol{b}} \mathrm{EtOH}(32 \mathrm{~mL})$ at $25^{\circ} \mathrm{C}$ for 2 days; ${ }^{\boldsymbol{c}}$ removal of the solvent by evaporation.

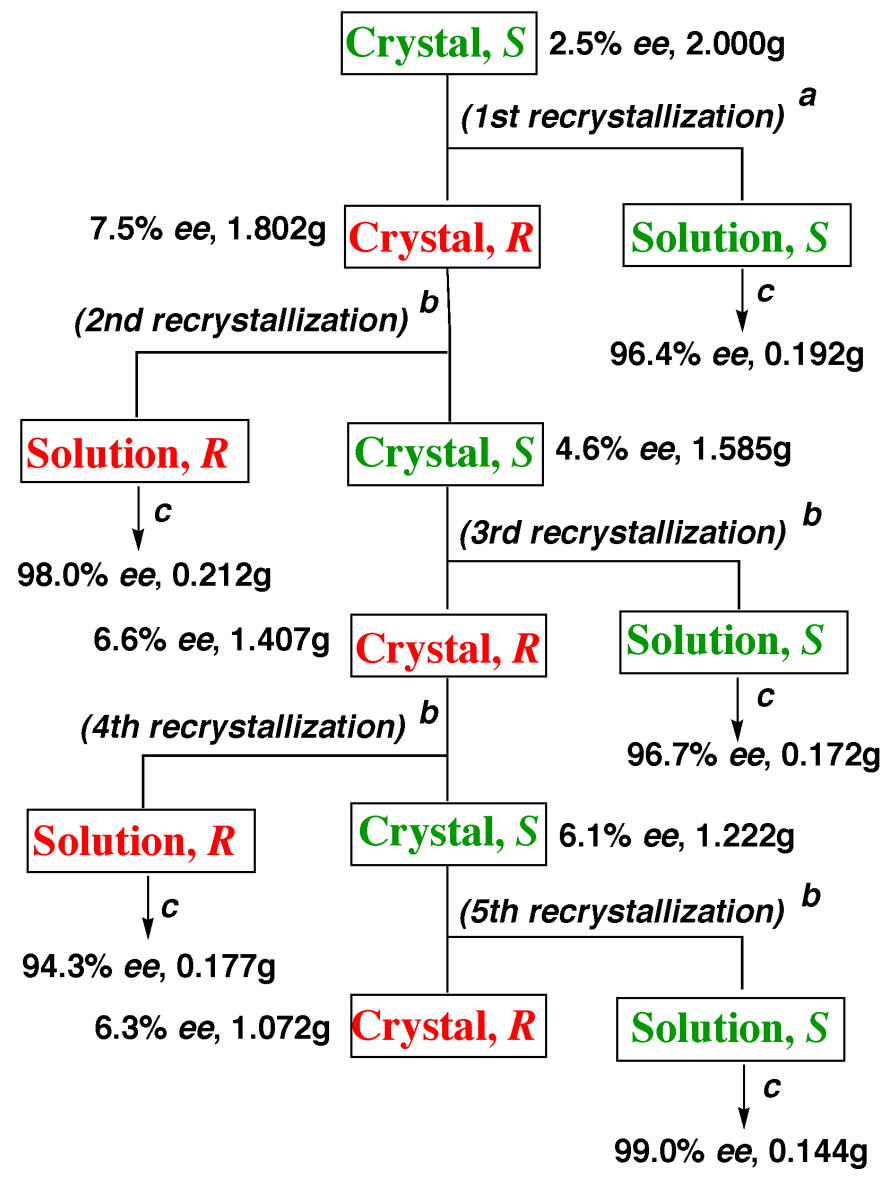


Figure 5. Molecular structure and crystal forms of the nonracemizable compounds showing preferential enrichment. Abbreviations of typical compounds are as follows: For $\mathrm{ST}, \mathrm{S}$ stands for sulfonium ion and T represents $p$-toluenesulfonate group. For $\mathrm{NNMe}_{3}$, the first $\mathrm{N}$ stands for ammonium ion, the second $\mathrm{N}$ represents $p$-nitorobenzenesulfonate group, and the last $\mathrm{Me}_{3}$ indicates trimethyl-substituted ammonium structure. For $\mathrm{NNMe}_{3}-\mathrm{OPh}$, $\mathrm{OPh}$ indicates the presence of a terminal phenoxy group in place of a terminal ethoxy group in $\mathrm{NNMe}_{3}$.
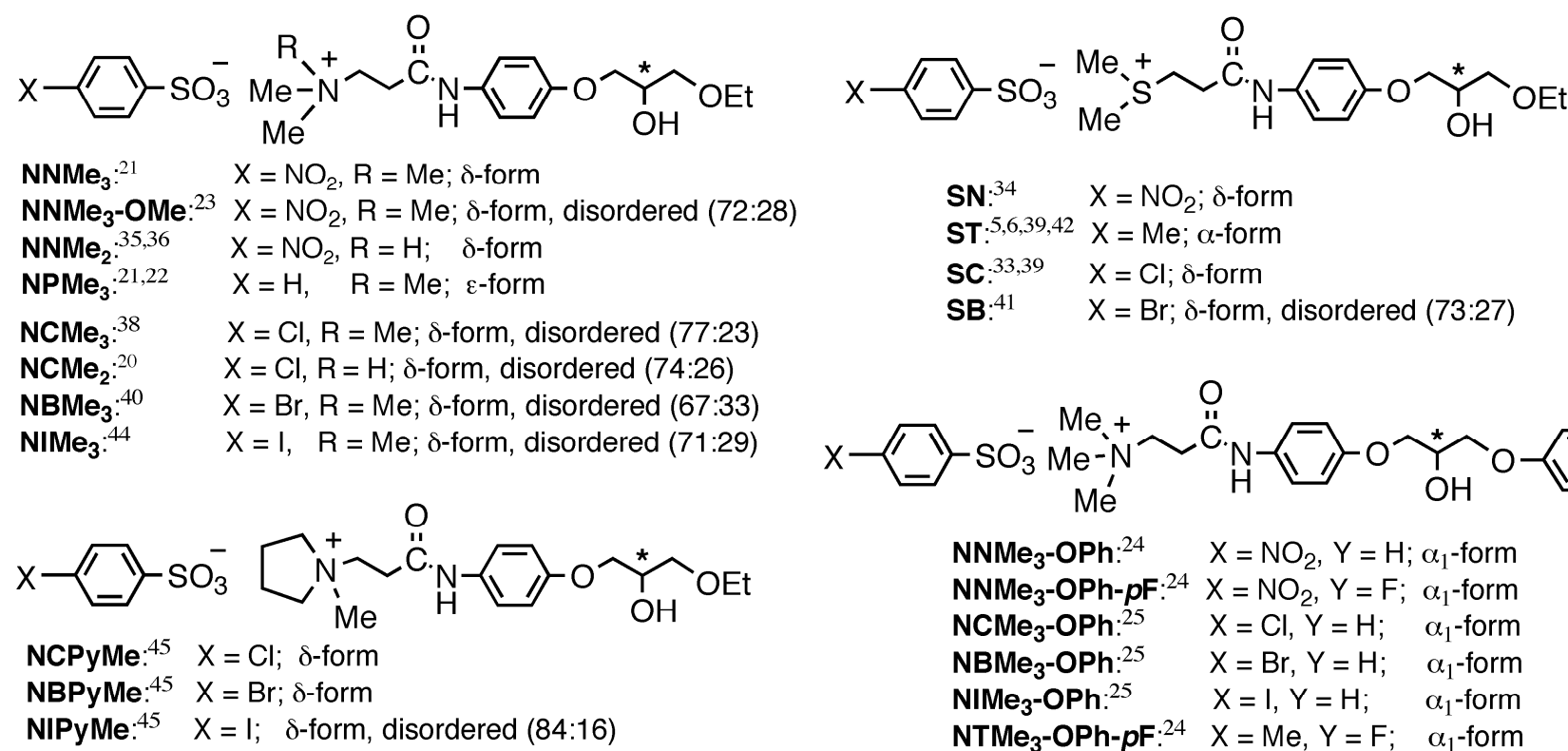

$\mathbf{S N}: 34 \quad X=\mathrm{NO}_{2} ; \delta$-form

ST:5,6,39,42 $X=\mathrm{Me} ; \alpha$-form

SC: $33,39 \quad X=C l ; \delta$-form

SB: ${ }^{41} \quad X=B r ; \delta$-form, disordered (73:27)

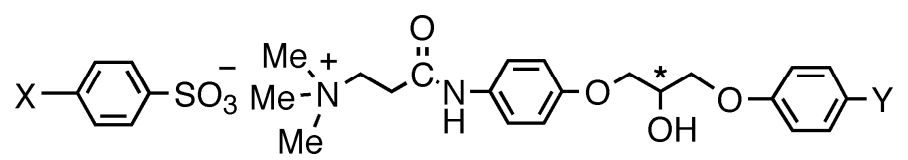

$\mathrm{NNMe}_{3}$-OPh: ${ }^{24} \quad \mathrm{X}=\mathrm{NO}_{2}, \mathrm{Y}=\mathrm{H} ; \alpha_{1}$-form

$\mathrm{NNMe}_{3}$-OPh-pF: ${ }^{24} \mathrm{X}=\mathrm{NO}_{2}, \mathrm{Y}=\mathrm{F} ; \alpha_{1}$-form

$\mathrm{NCMe}_{3}-\mathrm{OPh}:{ }^{25} \quad \mathrm{X}=\mathrm{Cl}, \mathrm{Y}=\mathrm{H} ; \quad \alpha_{1}$-form

NBMe $_{3}$-OPh: ${ }^{25} \quad \mathrm{X}=\mathrm{Br}, \mathrm{Y}=\mathrm{H} ; \quad \alpha_{1}$-form

NIMe $_{3}$-OPh: ${ }^{25} \quad X=I, Y=H ; \quad \alpha_{1}$-form

NTMe $_{3}$-OPh-pF: ${ }^{24} \quad X=$ Me, Y $=F ; \quad \alpha_{1}$-form

Accordingly, the technical and phenomenal features of preferential enrichment are summarized as follows $[7,8]$ :

1) Usual standard recrystallization conditions with neither vigorous stirring nor abrasive grinding are applied to the preferential enrichment experiment, except that approximately 4- to 25 -fold supersaturated solutions are employed because the supersolubility (a solubility obtained by dissolving the sample in a solvent on heating followed by being cooled) of the racemates showing preferential enrichment is considerably higher than that of the solubility at $25{ }^{\circ} \mathrm{C}$. The attainment of such a high supersolubility is closely associated with the preferential formation of homochiral 1D $R$ and $S$ chains even in the racemic solution. At the lower supersaturated concentrations, preferential enrichment does not efficiently occur.

2) Racemic or nonracemic samples of less than $10 \%$ ee are more suitable for the preferential enrichment experiment than those of higher $e e$ values to achieve a very efficient resolution.

3) Recrystallization of the nonracemic sample of less than $10 \%$ ee from the supersaturated solution leads to a remarkable enrichment of the excess enantiomer up to $100 \%$ ee in the mother liquors ( $a$ considerable enrichment of the excess enantiomer in the mother liquor). At the same time, the resulting deposited crystals always display the opposite chirality at around 5\% ee (a slight enrichment of the opposite enantiomer in the deposited crystals). These phenomena are fully reproducible. 
4) The solubility of the sample of a high ee value is much higher than that of a low ee one. This property allows dissolution of the excess enantiomer from the just-made crystals into solution until a slight enrichment of the opposite enantiomer in the deposited crystals occurs together with $a$ considerable enrichment of the excess enantiomer in the mother liquor.

5) When the original supersaturated solution is strictly racemic, the probability for either the $R$ or $S$ enantiomer to be enriched in the mother liquor after crystallization was $50 \%$. In the resulting deposited crystals, the opposite enantiomer is enriched up to around 5\% ee.

6) Only racemic or nonracemic samples have to be crystalline to implement the preferential enrichment experiment efficiently. It does not matter whether the enantiomerically enriched samples with high ee values exist as solids or oils, in sharp contrast to preferential crystallization of a racemic conglomerate.

7) Seed crystals are not necessary at all. Addition of seed crystals may accelerate or inhibit the occurrence of preferential enrichment, depending on the kind of added seed crystals.

8) The features of preferential enrichment are completely opposed to those of preferential crystallization of a racemic conglomerate, where a substantial enantiomeric enrichment does not occur in the mother liquor but in the deposited crystals.

\section{Mechanism of Preferential Enrichment}

In general, a polymorphic transition frequently occurs during crystallization from the supersaturated solutions of organic compounds, particularly when the packing mode in the first-formed crystal is metastable as the crystal structure [26-30]. Although much less is known about the mechanism of the polymorphic transition during crystallization from solution, it has been believed that the phase transition should proceed through either a solvent-mediated dissolution-recrystallization mechanism according to the 'Ostwald's law of stages'[31] or a solid-to-solid transformation one with the free energy change [32], and that the rate of the polymorphic transition primarily depends on the free energy difference between the two crystalline phases [26-30].

Thus far, we have found four types of solvent-assisted solid-to-solid transformations of a kinetically formed metastable $\gamma$-form crystalline phase into a thermodynamically more stable one $\left(\alpha-, \alpha_{1^{-}}, \delta_{-}\right.$, or $\varepsilon$-form) [5,6,20-25,33-45], which are relevant to the occurrence of preferential enrichment, in a process of crystal growth.

\subsection{Association Mode of Enantiomers in Solution}

To confirm the occurrence of the polymorphic transition and to elucidate the mechanism, it is primarily necessary to clarify the enantiomeric assembly mode in the first-formed metastable crystal prior to the polymorphic transition, and to compare it with the stable crystal structure after the polymorphic transition with respect to the racemic samples showing preferential enrichment. Since it is very possible that the stable molecular assembly structure in solution would be retained in the crystalline phase which is first formed by crystallization from the same solvent [46-48], we investigated the enantiomeric association mode in solutions of the racemates showing preferential enrichment. Although the variable temperature ${ }^{1} \mathrm{H}$ NMR technique proved to be inapplicable to deciding which molecular association mode is more stable in solution, homochiral or heterochiral 
[49-52], the combined use of solubility and supersolubility measurements under various conditions and number-averaged molecular weight measurement by vapor pressure osmometry turned out to be a potent tool for this objective [21]. Thus, it was concluded that a homochiral molecular assembly is essentially in preference to a heterochiral one in solutions of the racemic samples showing preferential enrichment, and that the homochiral supramolecular structure must be a $1 \mathrm{D}$ chain. These results were consistent with those obtained by the molecular dynamics simulation of the oligomer models of ST, $\mathrm{NNMe}_{3}$, and others (Figure 5) [21].

\subsection{Crystal Structures}

Figure 6. $\gamma$-Form crystal structure. (a) Intermolecular interactions and (b) schematic representation of the homochiral 1D chain. The ellipsoid and circle indicate the long-chain cation and sulfonate ion, respectively. The dashed lines show the intermolecular hydrogen bonds.

(a)

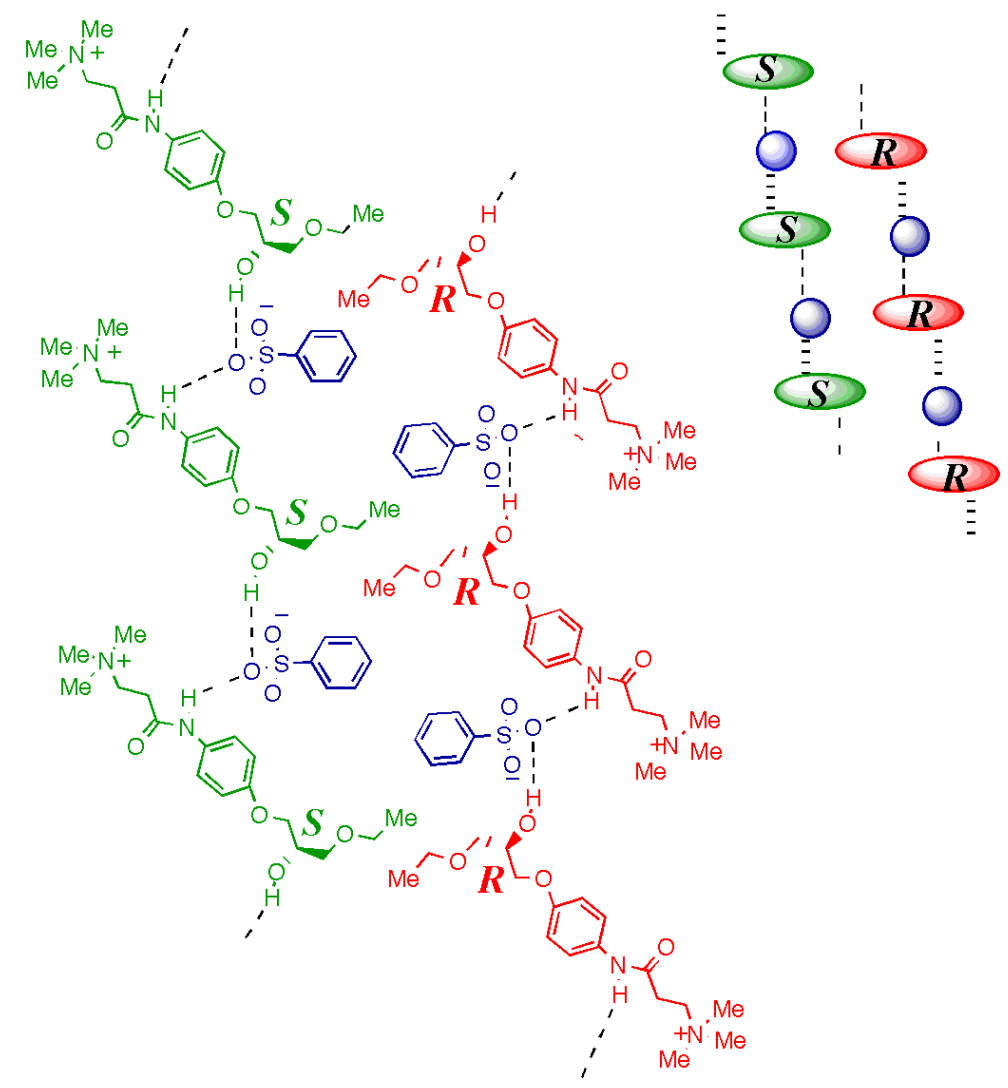

\subsubsection{Metastable $\gamma$-Form}

We have searched a compound that shows preferential enrichment and possesses a homochiral 1D chain structure in the metastable polymorphic form. As a result, the single crystal of racemic $\mathrm{NPMe}_{3}$ (Figure 5) was found to possess the desired $\gamma$-form crystal structure that is classified as a highly ordered racemic mixed crystal and is composed of alternating alignment of homochiral 1D $R$ and $S$ chains in an antiparallel direction with a space group $P-1(Z=2)$ with the second lowest symmetry; each homochiral chain comprises an alternating alignment of the long-chain ammonium ion and the 
sulfonate ion by two hydrogen bonds (i) between one oxygen atom of the sulfonate ion and the hydroxy group and (ii) between the same sulfonate oxygen atom and the amide NH (Figure 6) [21,22]. There is no interchain interaction. This $\gamma$-form crystal structure is also seen in several other racemic samples such as $\mathrm{NNMe}_{3}-\mathrm{OPr}, \mathrm{NBMe}_{3}-\mathrm{OPr}, \mathrm{NIMe}_{3}-\mathrm{OPr}$ and $\mathrm{NBMe}_{3}-\mathrm{OMe}$ which failed to show preferential enrichment $[21,23,44]$, indicating that the subsequent polymorphic transition into the more stable $\alpha-, \alpha_{1^{-}}, \delta$ - or $\varepsilon$-form is indispensable for preferential enrichment to occur. Actually, in the case of $\mathrm{NPMe}_{3}$, the successive polymorphic transition of the $\gamma$-form into the $\varepsilon$-form occurred slowly to induce preferential enrichment [21,22].

\subsubsection{Stable Forms}

Thus far four different types of stable crystal structures, $\alpha-, \alpha_{1^{-}}, \delta$ - and $\varepsilon$-forms, which are classified as a fairly or highly ordered racemic mixed crystal, have been obtained for the racemic or nearly racemic samples of the compounds showing preferential enrichment. Among them, the $\delta$-form and $\alpha_{1^{-}}$ form crystals are most commonly found for the compounds showing preferential enrichment [20-25,33-45], while only one case is observed for each of the $\alpha$ - and $\varepsilon$-forms [5,6,21,22,39]. In this section, the crystal structures of the $\delta$ - and $\alpha_{1}$-forms are described in detail, because this crystal structure provides a crucial information on the mechanism of the polymorphic transition causing preferential enrichment.

\section{(a) $\delta$-form}

In the $\delta$-form crystals of racemic and $S$-rich $(20 \% e e) \mathrm{NBMe}_{3}$ [40], the orientational disorder was observed at the position of the hydroxy group on an asymmetric carbon atom (Figure 7). The degree of disorder per asymmetric unit (one salt) for the racemic or $S$-rich sample was estimated to be 67:33 or 75:25 ( $R$ vs. $S$, or $S$ vs. $R$ ), respectively, by calculating the constrained occupancy factors of the hydroxy group, corresponding to a fairly ordered racemic mixed crystal. The $\delta$-form crystal structure of racemic $\mathrm{NBMe}_{3}$ is characterized by two types of cyclic dimers, types A and B; the head-to-head cyclic dimer of type A is formed by the hydrogen bonds between the hydroxy groups and the ethoxy oxygen atoms in a pair of $R$ and $S$ molecules, and another head-to-head cyclic dimer of type B is formed by i) the hydrogen bond between one oxygen atom of a sulfonate ion and the amide $\mathrm{NH}$ and ii) the electrostatic interaction between another oxygen atom of the same sulfonate ion and the ammonium nitrogen atom in the neighboring long-chain cation (Figure 7). By virtue of these intermolecular interactions, a heterochiral 1D chain is formed. Furthermore, each 1D chain interacts with two adjacent chains by another weak electrostatic interaction between the third oxygen atom of the same sulfonate ion and the ammonium nitrogen atom in the adjacent chains, eventually forming a weak 2D sheet structure. This fragile 2D sheet structure is essential for crystal disintegration resulting in preferential enrichment as discussed in section 3.4.

Furthermore, the crystal structure of the $S$-rich $(20 \%$ ee $) \mathrm{NBMe}_{3}$ was virtually isostructural with that of the racemate and very similar to that of the pure $S$ enantiomer (Figure 7) [40]. 
Figure 7. $\delta$-From crystal structures of racemic $(0 \% e e)$ and $S$-rich $(20 \% e e) \mathrm{NBMe}_{3}$. (a) Intermolecular interactions and (b) schematic representation of the heterochiral cyclic dimer and the homochiral noncyclic ones in the crystal. The ellipsoid and circle indicate the long-chain ammonium ion and sulfonate ion, respectively. The dashed lines show the intermolecular hydrogen bonds and electrostatic interactions. The hydroxy group on the asymmetric carbon atom is disordered over two positions in panel a. The $R$ and $S$ enantiomers in the sites with higher occupancy factor $(0.67$ or 0.75$)$ are designated $\boldsymbol{R}$ and $\boldsymbol{S}$, and those in the sites with lower occupancy factors $(0.33$ or 0.25$)$ are $\boldsymbol{r}$ and $\boldsymbol{s}$, for racemic $(0 \% e e)$ and $S$-rich $(20 \% e e)$ crystal, respectively. The contents of three dimer structures were estimated from the occupancy factors of the orientationally disordered hydroxy groups and the ee value of the crystal.

(a)

(b)
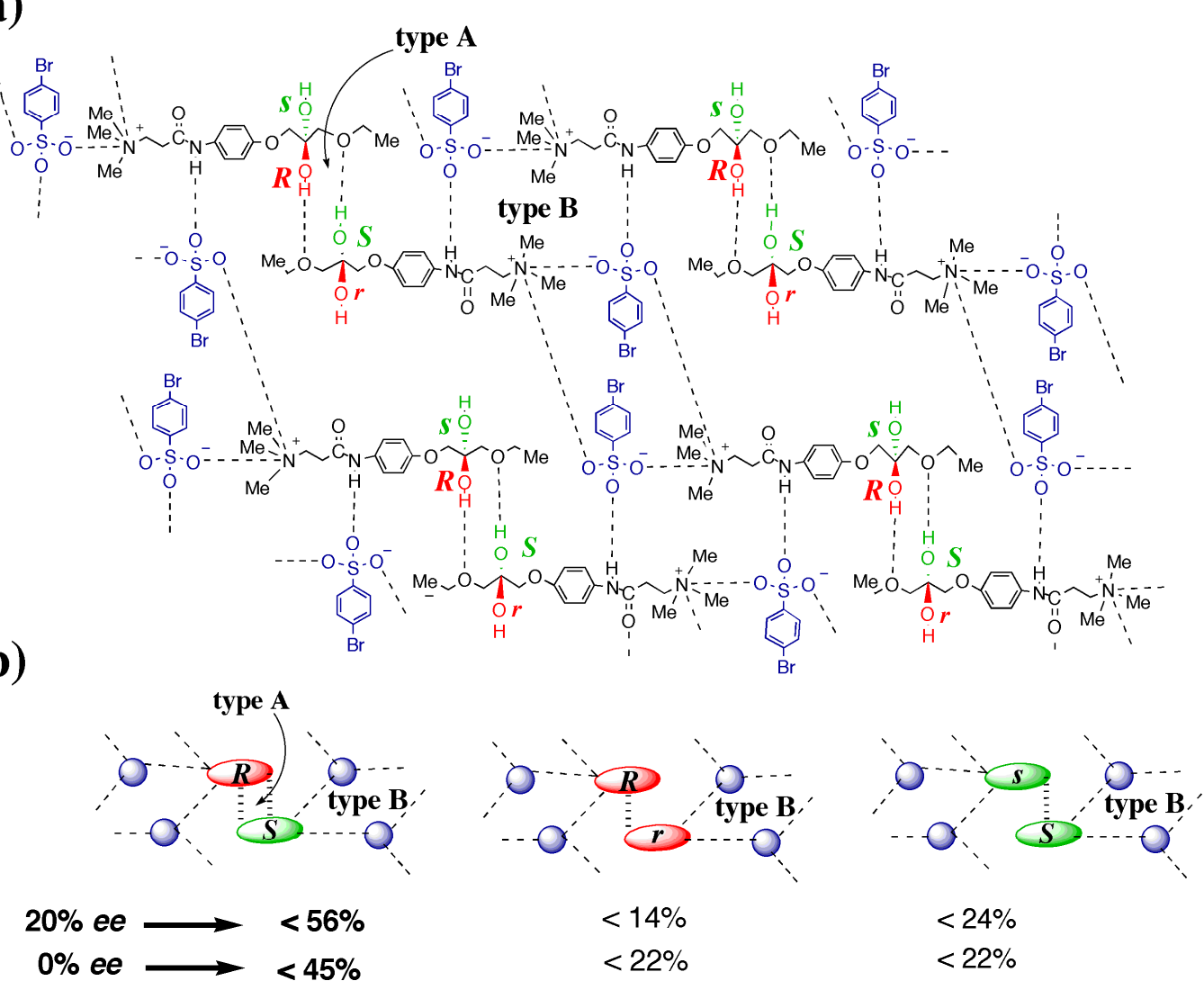

(b) $\alpha_{1}$-form

The $\alpha_{1}$-form crystals were formed for the compounds such as $\mathrm{NBMe}_{3}-\mathrm{OPh}$ that have a terminal phenoxy group (Figure 5) [24,25]. This $\alpha_{1}$-form crystal structure is characterized by a heterochiral 1D chain which consists of two types of cyclic dimers (type $\mathrm{B}$ and type $\mathrm{C}$ ); the type $\mathrm{C}$ is formed by the hydrogen bonds between two hydroxy groups and two carbonyl oxygen atoms, while the type B is similar to that in the $\delta$-form crystal. Furthermore, each 1D chain interacts with two adjacent chains by two interactions forming a weak $2 \mathrm{D}$ sheet structure; one is the slipped-parallel $\pi-\pi$ stacking between the benzene rings of the nearest terminal phenoxy groups in a pair of $R$ and $S$ enantiomers, and the other is the phenyl centroid $\cdots \mathrm{Br}-\mathrm{C}\left(\mathrm{sp}^{2}\right)$ Coulombic donor-acceptor interchain interactions between the bromine atom of the $p$-bromobenzenesulfonate ion and the internal benzene ring of the neighboring 
long-chain cation (Figure 8). This fragile 2D sheet structure is essential for crystal disintegration resulting in preferential enrichment, too.

Figure 8. $\alpha_{1}$-Form crystal structure. (a) Crystal structure of racemic $\mathrm{NBMe}_{3}-\mathrm{OPh}$ and (b) schematic representation of the intermolecular interactions.

(a)

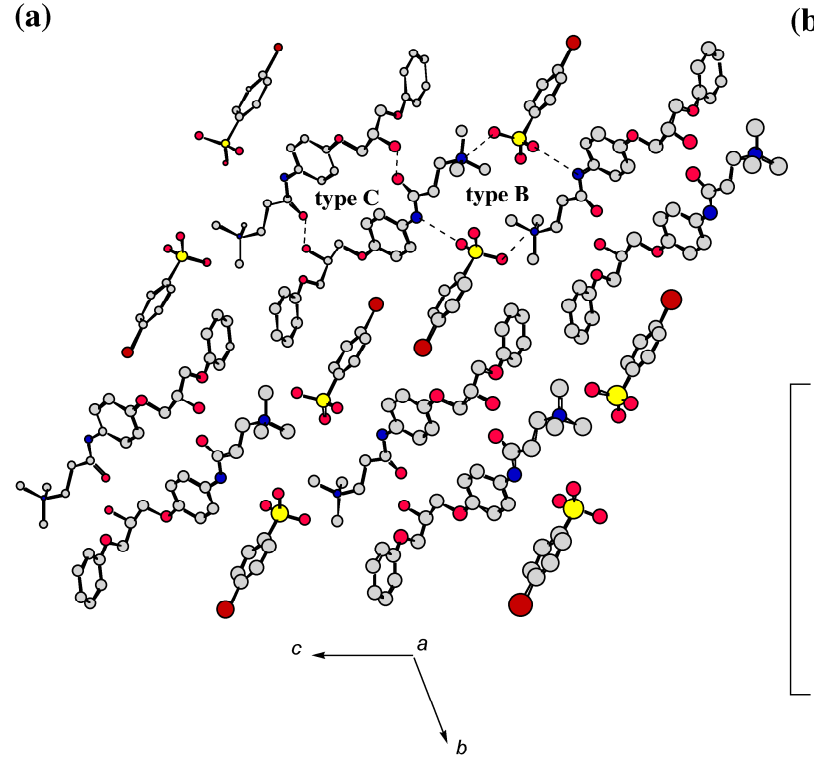

(b)

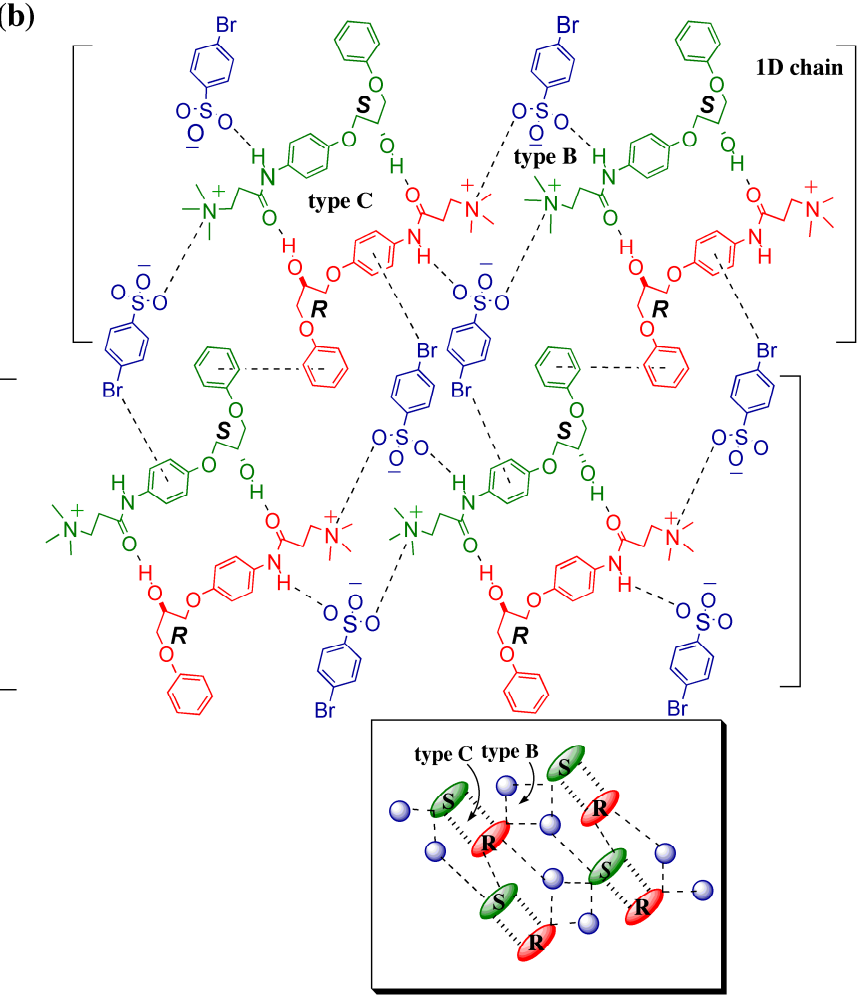

\subsection{Melting Point Diagram}

Two kinds of unique melting point diagrams obtained experimentally for two typical compounds showing preferential enrichment, $\mathrm{NNMe}_{3}$ and $\mathrm{NBMe}_{3}$, deserve attention.

In the case of $\mathrm{NNMe}_{3}$, two curves were found to intersect at two points around 35\% ee (Figure 9a). The X-ray crystallographic analyses indicated that the curve in the range of 0-15\% ee corresponds to $a$ highly or fairly ordered racemic mixed crystal [21]. On the other hand, the overall flat curve in the range of more than $40 \%$ ee proved to correspond to a less ordered racemic mixed crystal composed of different amounts of the $R$ and $S$ enantiomers in the crystal lattice.

In the case of $\mathrm{NBMe}_{3}$, there are two convex curves which do not intersect but are located closely to each other (Figure 9b); the upper one proved to belong to a fairly ordered racemic mixed crystal by X-ray crystallographic analyses [40], and the other corresponds to a metastable crystalline phase that suddenly disappeared prior to its characterization and was never observed again [40].

From these diagrams, the following three possibilities can be suggested; i) their crystalline nature falls into a racemic mixed crystal, ii) there must be a polymorphism in both cases, and iii) the free energy difference between the two polymorphs is small enough to allow a polymorphic transition to proceed at a moderate rate during crystallization. 
Figure 9. Melting point diagrams. (a) $\mathrm{NNMe}_{3}$ and (b) $\mathrm{NBMe}_{3}$. White and black circles represent the temperatures of the beginning and the end of fusion, respectively.

(a)

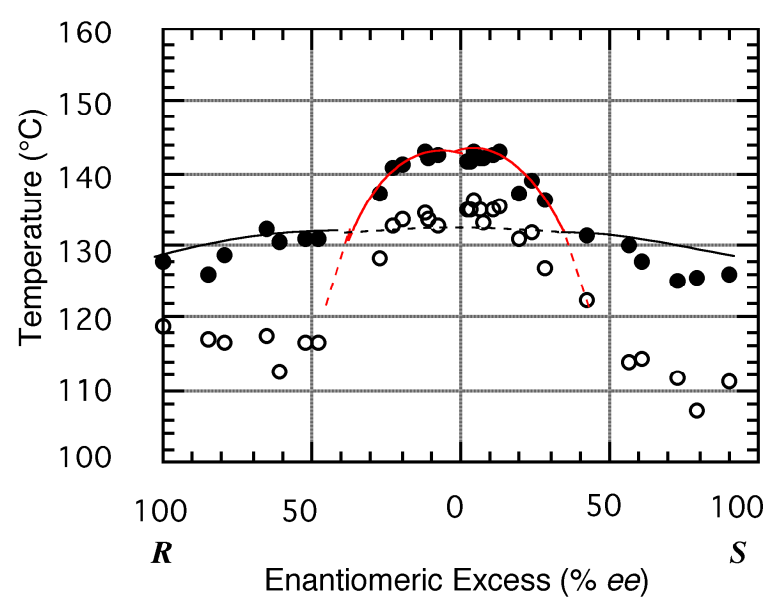

(b)

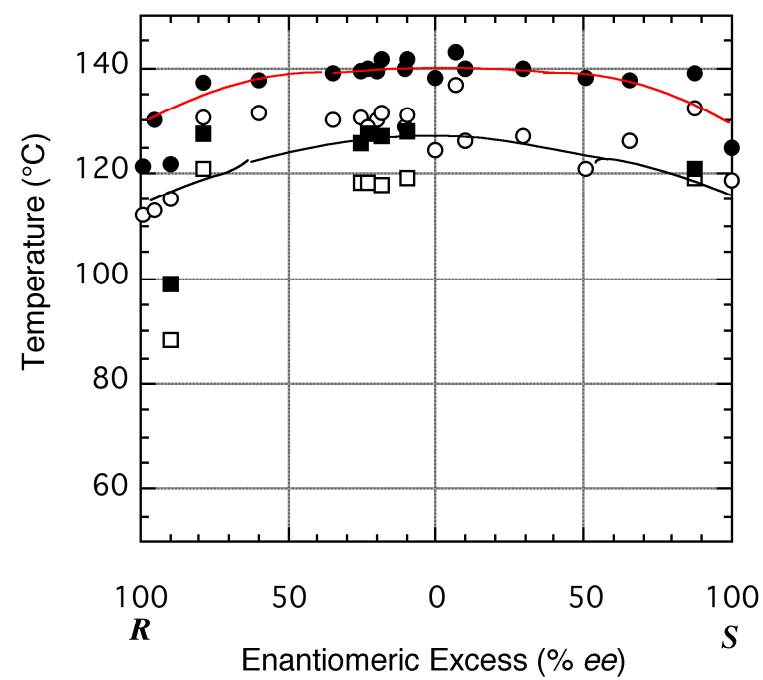

\subsection{Flexible Modes of Polymorphic Transition}

\subsection{1. $\gamma$ to $\delta$ transition}

Before discussing the mode of the polymorphic transition, let's consider the association mode of the homochiral 1D $R$ and $S$ chains in the metastable $\gamma$-form crystal formed kinetically from the highly supersaturated solution (Figure 10). Supposed that the $R$ enantiomer is slightly excess in the supersaturated solution, the resulting metastable $\gamma$-form crystal must become $R$-rich, too. This slightly $R$-rich metastable crystal exists as a fairly ordered racemic mixed crystal which consists of three types of alignment modes of the homochiral $R$ and $S$ chains; the major regular alignment of the $R$ and $S$ chains, along with two minor irregular alignments in which an odd number of $R$ chains or an even number of $R$ ones are surrounded by two $S$ chains (Figure 10a).

Now let's consider the mode of the polymorphic transition of the metastable $\gamma$-form into the $\delta$-form. By comparing the crystal structures between the metastable $\gamma$-form composed of the homochiral 1D $R$ and $S$ chains and the stable $\delta$-form comprised of the heterochiral 2D sheet, the mode of this single phase transition can be proposed (Figure 10b).

Polymorphic transition in the metastable $\gamma$-form crystal is initiated by rearrangement of the hydrogen bonds inside the crystal lattice. It is very possible for the nearest $R$ and $S$ molecules in the two adjacent chains in the $\gamma$-form to form new hydrogen bonds between the ethoxy oxygen atoms and the hydroxy groups by slight movement of the two molecules in the crystal. This rearrangement of hydrogen bonds, accompanied by slight movement of the sulfonate ions so as to form the cyclic dimers of types A and B, occurs one after another in the crystal lattice to lead to the heterochiral 1D chains and then the weak 2D sheet structure (Figures 11-13). 
Figure 10. Mechanism of preferential enrichment. (a) Association mode of homochiral 1D $R$ and $S$ chains in the metastable $\gamma$-form crystal formed kinetically from the highly supersaturated solution of slightly $R$-rich sample. (b) Modes of polymorphic transition of the $\gamma$-form into the $\delta$-form in the regular and irregular alignment areas of the $R$ and $S$ chains in the crystal.

(a)

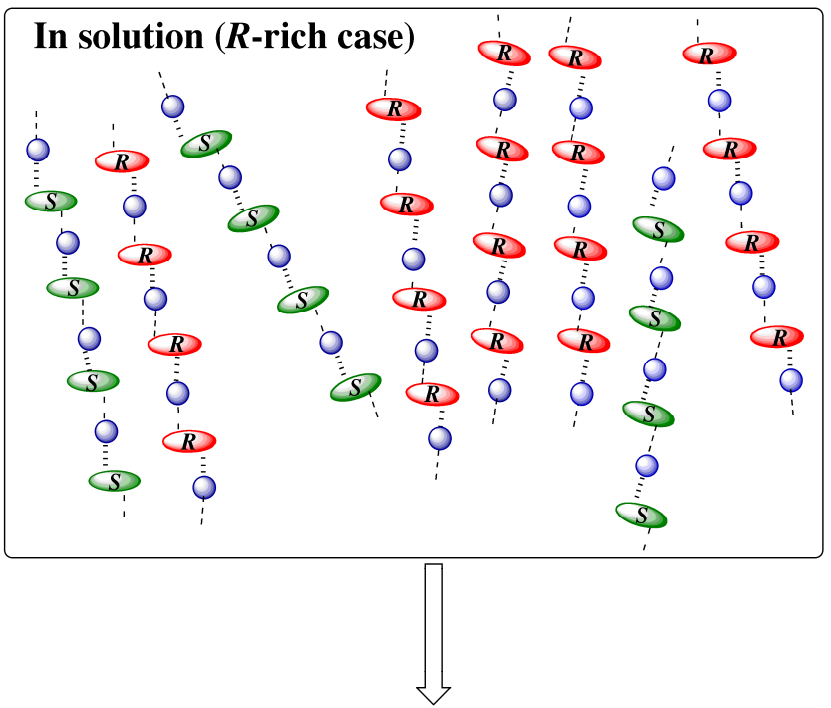

\section{Metastable $\gamma$-form crystal ( $R$-rich)}
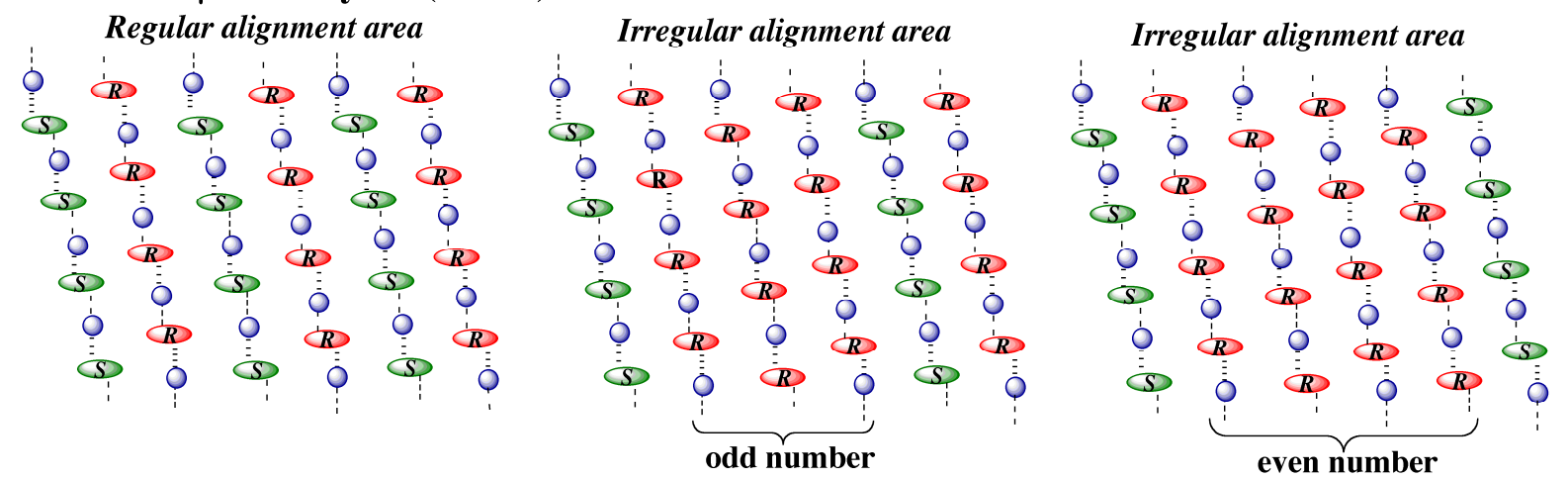

(b)

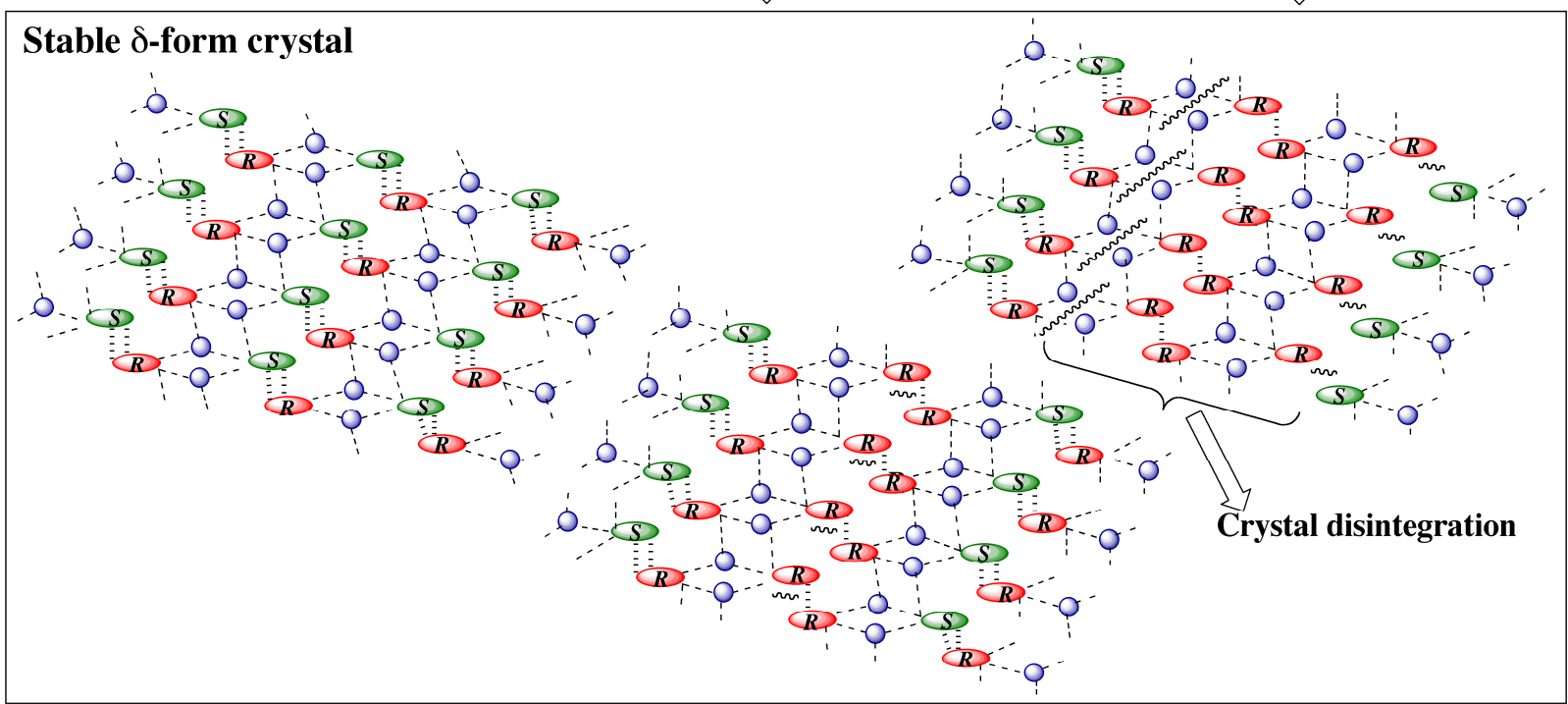


The fashion of alignment of the homochiral $R$ and $S$ chains in the metastable $\gamma$-form crystal must define the type of the local $\delta$-form crystal structure after the phase transition:

1) At the sites where equal numbers of homochiral $R$ and $S$ chains are alternately aligned in an antiparallel direction along one axis, a highly ordered $\delta$ form crystal structure will be formed locally after the polymorphic transition (Figures $10 \mathrm{~b}$ and 11).

2) At the sites where an odd number of homochiral $R$ chains are aligned between two $S$ chains, after the polymorphic transition, the disordered $\delta$-form crystal structure will be formed locally without disintegration of the crystal (Figures $10 \mathrm{~b}$ and 12).

3) At the sites where an even number of homochiral $R$ chains are aligned between two $S$ chains, after the polymorphic transition, partial crystal disintegration occurs in the resulting $\delta$-form crystal to release the $R$-rich area into solution, because the $R$-rich area was surrounded by the sites where the $\mathrm{OH} \cdots \mathrm{OEt}$ interaction cannot be formed at all as well as the sites with weak electrostatic interactions (Figures $10 \mathrm{~b}$ and 13).

This third case corresponds to the redissolution of the excess enantiomer from the just-made crystals into solution and must be responsible for the phenomenon characteristic of preferential enrichment, a considerable enrichment of the excess enantiomer in the mother liquor until a slight enrichment of the opposite enantiomer in the deposited crystals occurs.

Figure 11. Polymorphic transition in the case where equal numbers of homochiral $R$ and $S$ chains are alternately aligned in the $\gamma$-form crystal, leading to the ordered $\delta$-form crystal.

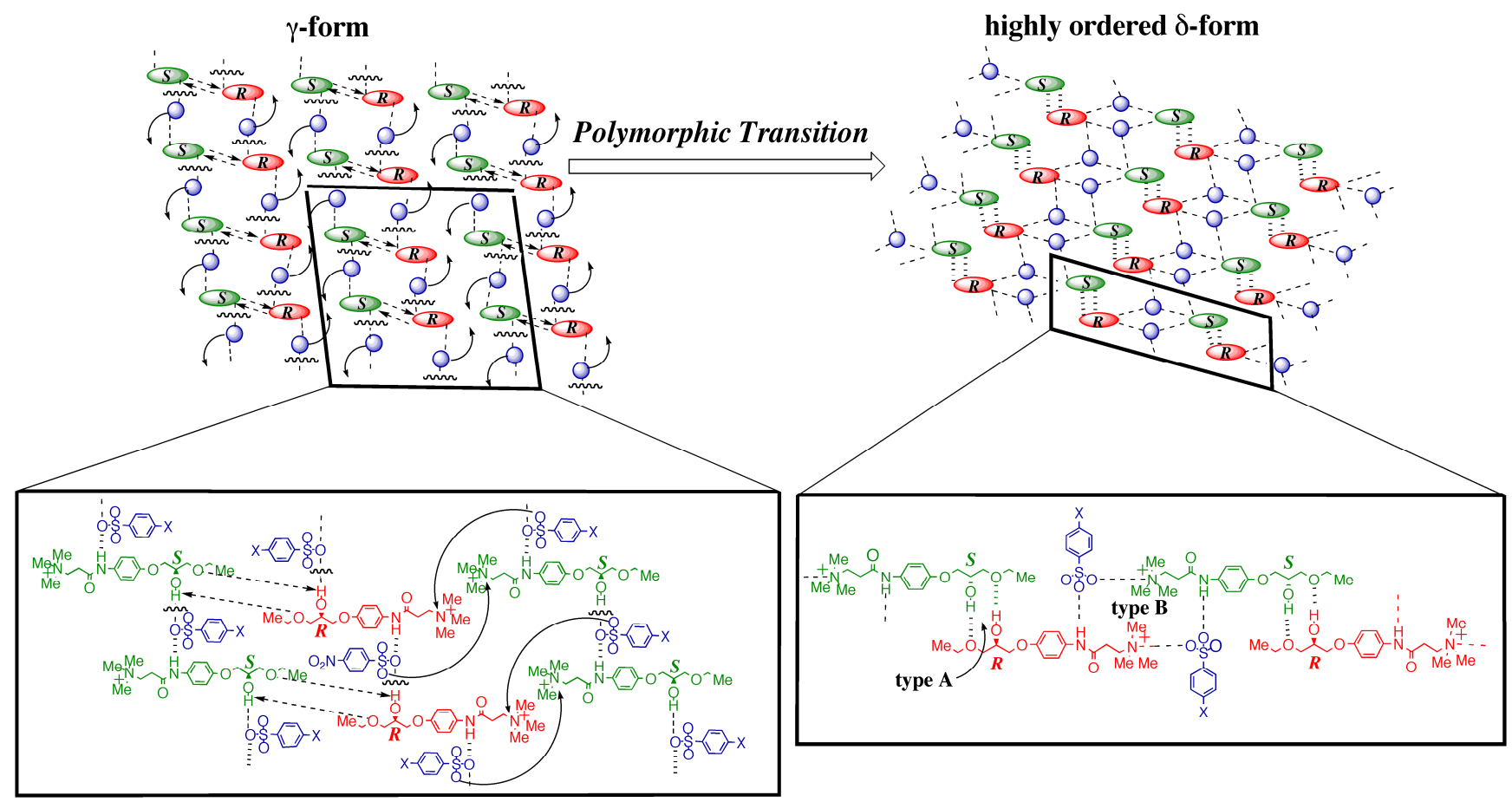


Figure 12. Polymorphic transition in the case where an odd number of homochiral $R$ chains are surrounded by two $S$ chains in the $\gamma$-form crystal, leading to the fairly disordered $\delta$-firm crystal without crystal disintegration.

$\gamma$-form

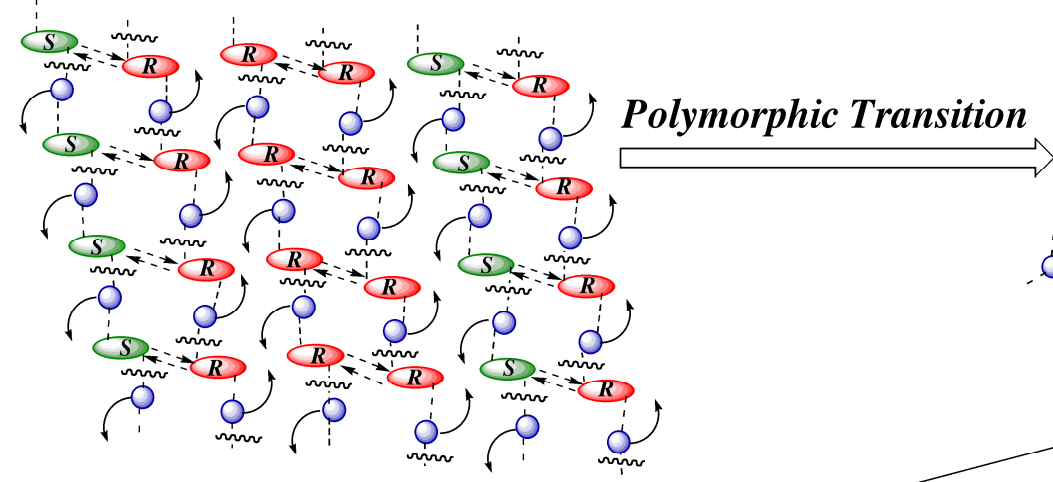

fairly ordered $\delta$-form

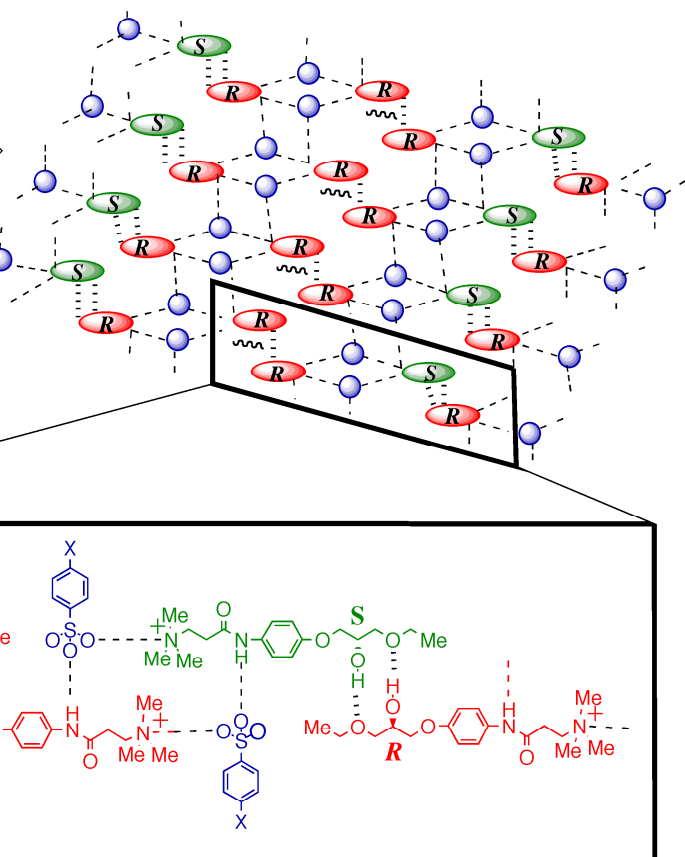

Figure 13. Polymorphic transition in the case where an even number of homochiral $R$ chains are surrounded by two $S$ chains in the $\gamma$-form crystal, leading to local crystal disintegration in the $\delta$-form crystal that occurs between the sites $a$ and $d$.

$\gamma$-form

$\delta$-form

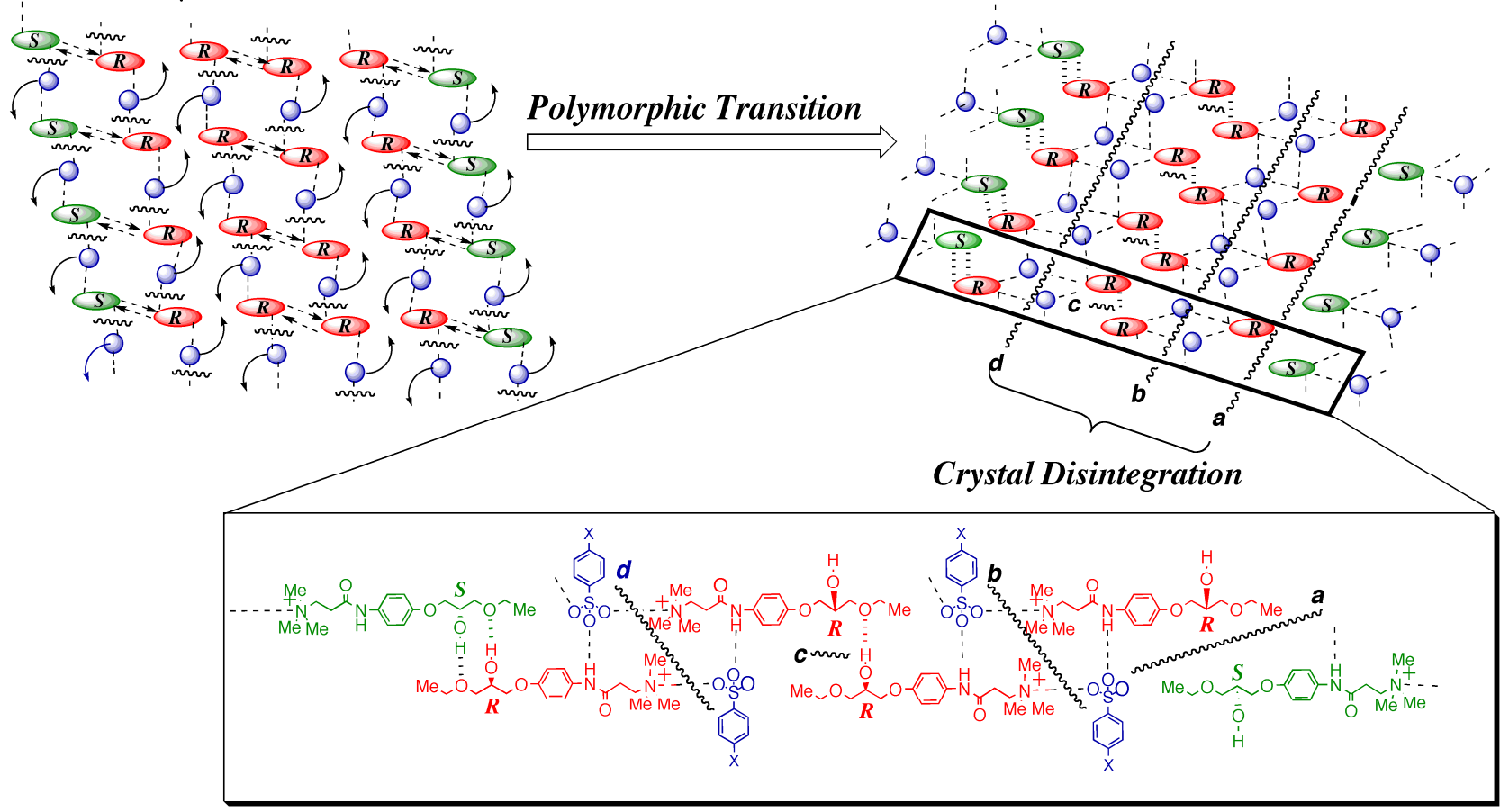




\subsection{2. $\gamma$ to $\alpha_{1}$ transition}

Figure 14. The mode of polymorphic transition. From (a) $\gamma$-form to (b) $\delta$-form or (c) $\alpha_{1^{-}}$ form.

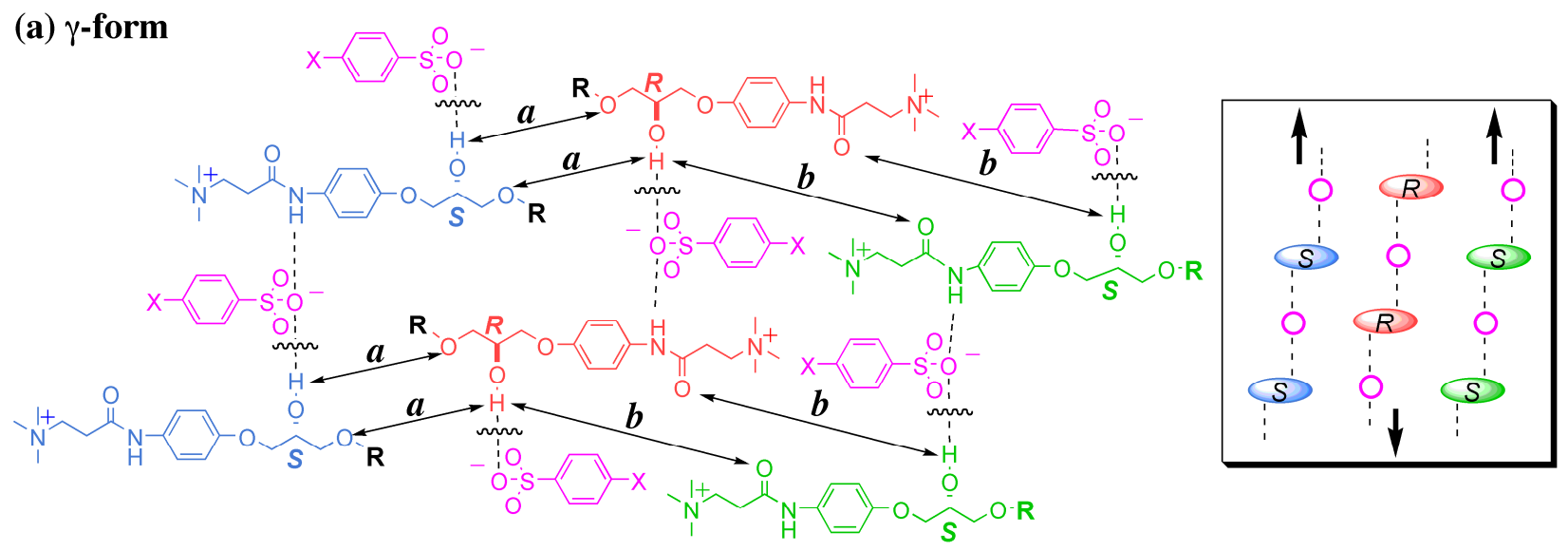

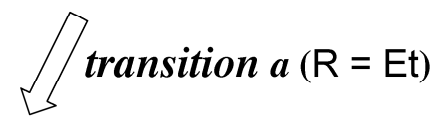

(b) $\delta$-form

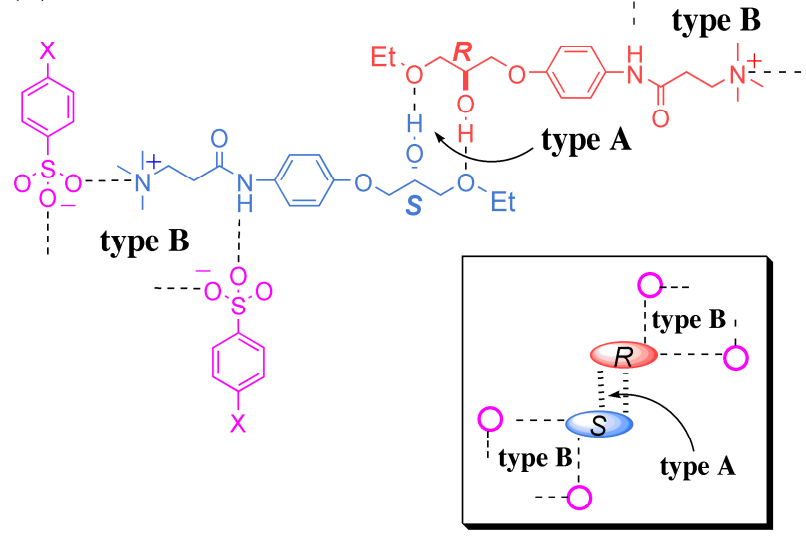

$\sum_{\text {transition } b}(\mathrm{R}=\mathrm{Ph})$

(c) $\alpha_{1}$-form

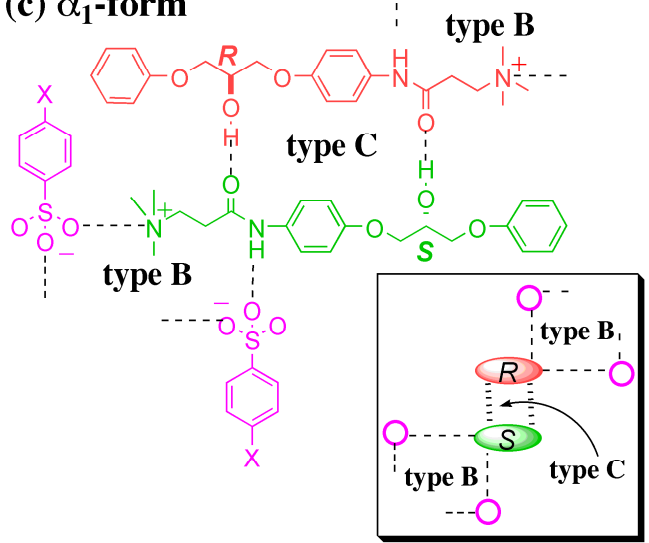

On the analogy of the mode of the polymorphic transition of the $\gamma$-form into the $\delta$-form, we propose the mode of $\gamma$-to- $\alpha_{1}$ polymorphic transition (Figure 14) [24,25]. Polymorphic transition is initiated by rearrangement of the hydrogen bonds inside the $\gamma$-form crystal lattice. The nearest $R$ and $S$ molecules in the two adjacent chains in the $\gamma$-form form new hydrogen bonds between the hydroxy group and the carbonyl oxygen atom by slight movement of the two molecules inside the crystal lattice to give a cyclic dimer of type $\mathrm{C}$ (Figure 14c). This molecular movement occurs in an opposite direction to the case of the $\gamma$-to- $\delta$ transition, where new hydrogen bonds are formed between the hydroxy groups and the alkoxy oxygen atoms to give a cyclic dimer of type A (Figure 14b) [21,40]. Namely, the terminal phenoxy group prevents the $\gamma$-to- $\delta$ transition $\boldsymbol{a}$ due to the steric hindrance, instead the $\gamma$-to- $\alpha_{1}$ transition $\boldsymbol{b}$ occurs. Subsequently, this rearrangement of hydrogen bonds, accompanied by slight movement of the phenylsulfonate groups so as to form cyclic dimers of types B and C, occurs one after another in the crystal lattice and leads to the heterochiral 1D chains and then the weak 2D sheet structure (Figure 8). In an area where an even number of homochiral $S(R)$ chains are surrounded by two $R(S)$ chains in 
the $\gamma$-form crystal, partial crystal disintegration occurs after polymorphic transition, resulting in the occurrence of preferential enrichment (Figure 15).

Figure 15. Polymorphic transition of the metastable $\gamma$-form into the stable $\alpha_{1}$-form. This is a case in which an even number (four in this case) of homochiral $S$ chains are surrounded by two $R$ chains in the $\gamma$-form crystal, resulting in partial crystal disintegration after polymorphic transition; three right-side $S$ chains which cannot form a complete type C cyclic dimer dissolve into solution, while one left-side $S$ chain remains by forming the type C cyclic dimer.

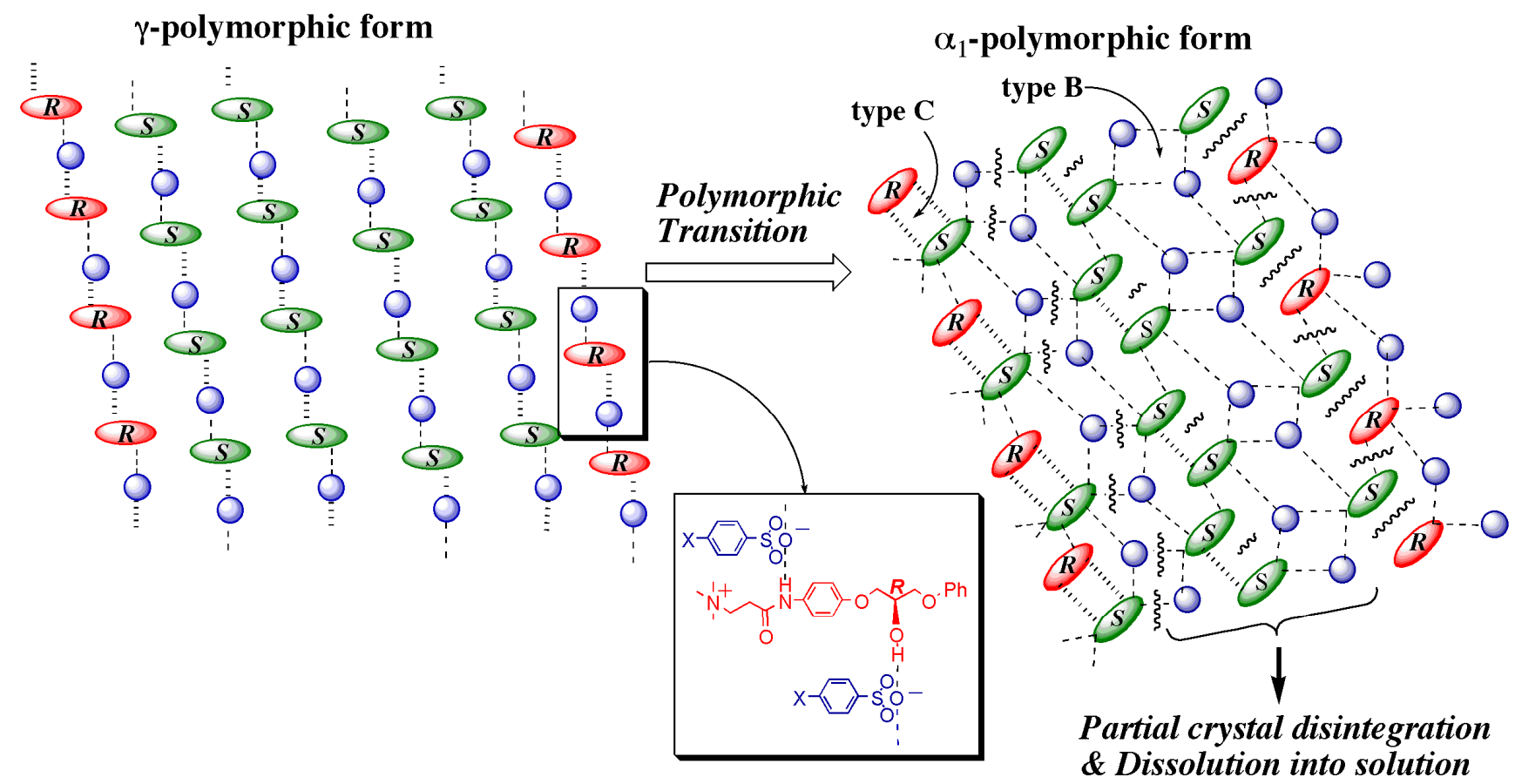

\subsection{Overall Mechanism of Preferential Enrichment}

The origin of preferential enrichment can be interpreted in terms of an interplay of i) the polymorphic transition of the metastable $\gamma$-form into the stable $\alpha-, \alpha_{1^{-}}, \delta$-, or $\varepsilon$-form, ii) the substantially higher solubility of the sample with high $e e$ values than that of low ee ones resulting in $a$ considerable enrichment of the excess enantiomer in the mother liquor until a slight enrichment of the opposite enantiomer in the deposited crystals occurs.

The proposed overall mechanism of preferential enrichment illustrated in Figure 10 is the case that a considerable enrichment of the $R$ enantiomer occurs in the mother liquor by crystallization from the slightly $R$-rich (around 5\% ee) supersaturated solution. In solution, homochiral 1D $R$ and $S$ chains are formed preferentially and aggregate to form a $\gamma$-form supramolecular cluster, which undergoes a phase transition to give the incipient metastable $\gamma$-form crystals with fair disorder. Since the $R$ enantiomer is slightly in excess in solution, the resulting metastable $\gamma$-form crystals become slightly $R$-rich, too. This 
slightly $R$-rich crystals consist of three types of alignment modes of the homochiral $R$ and $S$ chains; the major regular alignment of the $R$ and $S$ chains, along with two minor irregular alignments in which an odd number of $R$ chains or an even number of $R$ ones are surrounded by two $S$ chains. The next step is the polymorphic transition initiated by the rearrangement of the hydrogen bonds in the crystal lattice as discussed in the section 3.4. After the polymorphic transition, partial crystal disintegration in the resulting $\delta$-form crystals occurs at the sites where an even number of homochiral $R$ chains are aligned between two $S$ chains, releasing the $R$-rich area into solution. In contrast, at the sites where an even number of homochiral $S$ chains are aligned between two $R$ chains, the dissolution of the $S$-rich area into solution seemingly does not proceed; as soon as the dissolved $S$ enantiomers form 1D $S$ chains, they meet with excess 1D $R$ chains in solution, and then crystallization of the $R$-rich $\gamma$-form crystals and the subsequent polymorphic transition into the $\delta$-form occur, followed by redissolution of the excess $R$ enantiomer into solution to result in the eventual deposition of slightly $S$-rich $\delta$-form crystals. Thus, the ee value of the $R$ enantiomer in the mother liquor is gradually raised until the opposite $S$ enantiomer becomes in a small excess in the deposited crystals.

It should be stressed again that when the original supersaturated solution was strictly racemic $(0.0 \% e e)$, the probability for either the $R$ or the $S$ enantiomer to be enriched in solution after crystallization was 50\%; this is because initial capricious formation of the very first nonracemic metastable $\gamma$-form crystal nucleus should doom which enantiomer is enriched in the mother liquor, $i$. e., this is a typical feature in an event of complexity.

\subsection{Requirements for the Occurrence of Preferential Enrichment}

From the mechanistic studies described above, it is concluded that the following three requirements have to be satisfied to induce preferential enrichment for a racemic sample:

1) Polymorphic transition of a metastable polymorphic form into a stable one must occur during crystallization; this process is the essence of chiral symmetry breaking. This polymorphic transition can be monitored by the in situ ATR-IR spectroscopy. If there is a distinct difference in the vibrations of hydrogen bond forming groups between the supersaturated solution before crystallization and the deposited crystals after crystallization, polymorphic transition is most likely to occur.

2) The solubility of the enantiomerically pure sample is much higher than that of the corresponding racemic sample; this phenomenon is closely related to the preferential formation of homochiral 1D chain even in the racemic solution. The difference in the solubility between the racemic sample and the enantiomerically enriched one substantially affect the ee value reached in the mother liquor after crystallization; the larger the difference is, the higher the reached $e e$ value is.

3) The deposited crystals must be a mixed crystal composed of fairly random alignment of two enantiomers so that the chiral symmetry breaking can be memorized.

A convenient method to judge whether preferential enrichment occurs or not for a given sample is to repeat recrystallization of the nonracemic sample adjusted to 5 to $10 \%$ ee at 25,0 , or $-20^{\circ} \mathrm{C}$ at several different supersaturated concentrations between 5- to 20-fold and measure the ee value in the mother liquor by HPLC analysis, etc. after each crystallization. 


\section{Induction of Preferential Enrichment}

Preferential enrichment is strongly affected by the surrounding conditions, such as additives (seed crystals), solvent, concentration, and temperature, as well as the molecular structure [7,8]. Accordingly, both induction and inhibition of preferential enrichment were accomplished by controlling the mode of the polymorphic transition during crystallization with appropriate seed crystals, on the basis of the "epitaxial transition" phenomenon that consists of 1) the adsorption of $\gamma$-form prenucleation aggregates, 2) the heterogeneous nucleation and crystal growth of a metastable $\gamma$-form polymorph, and 3) the subsequent polymorphic transition into the more stable ones such as $\delta$ - and $\alpha_{1}$-forms, on the same surface of a seed crystal (Figure 16) [23,24].

Figure 16. Schematic representation of epitaxial transition of a metastable $\gamma$-form polymorph into an $\alpha_{1}$-form one on the surface of an $\alpha_{1}$-form seed crystal, inducing preferential enrichment.
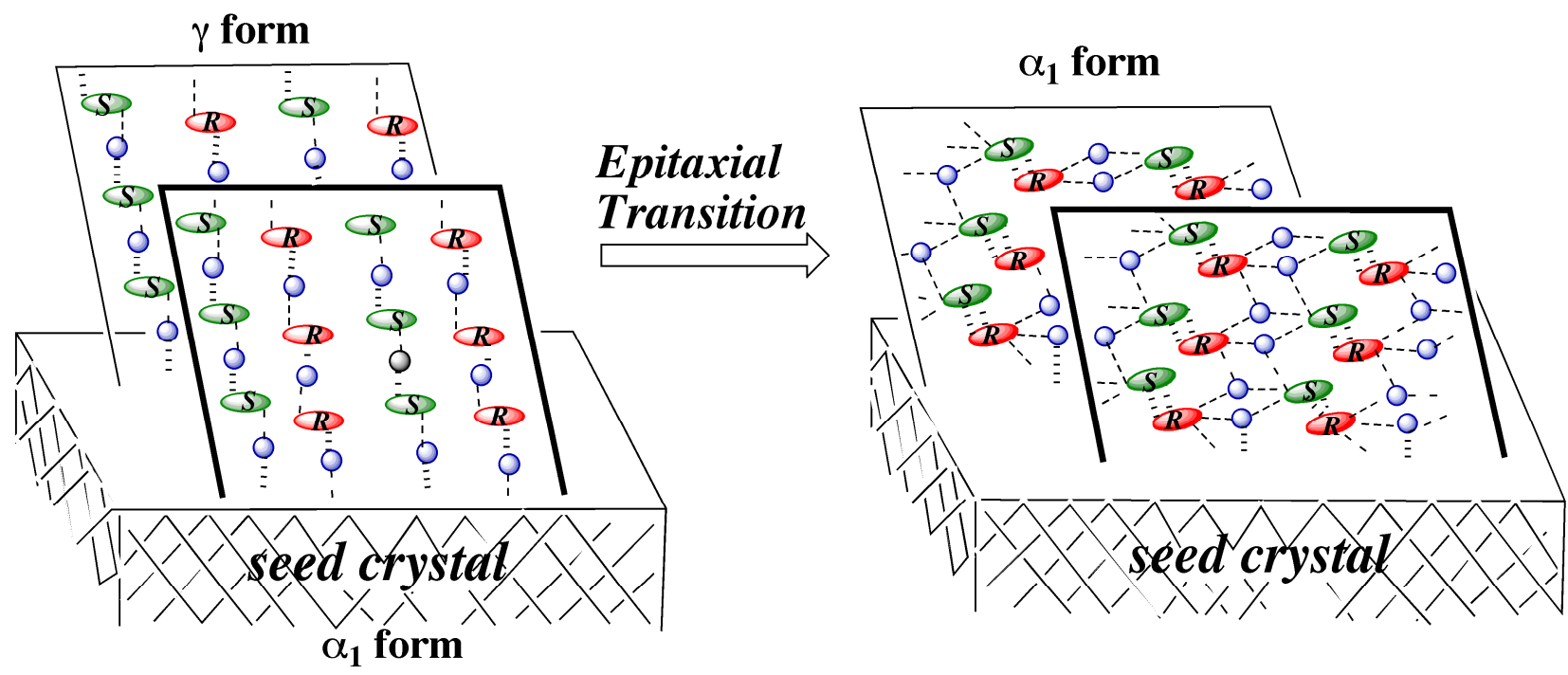

As a typical example of induction, addition of the $\alpha_{1}$-form seed crystals of $( \pm)-\mathrm{NTMe}_{3}-\mathrm{OPh}-p \mathrm{~F}$ (Figure 5), which can easily show preferential enrichment, to the supersaturated EtOH solution of $( \pm)$ $\mathrm{NTMe}_{3}-\mathrm{OPh}$, which does not show this phenomenon by itself due to the undesired polymorphic transition of the $\gamma$-form into the more stable $\mu$-form, can explicitly induce the preferential enrichment of ( \pm )-NTMe $\mathrm{N}_{3}-\mathrm{OPh}$, accompanied by the deposition of the $\alpha_{1}$-form crystals of $( \pm)-\mathrm{NTMe}_{3}-\mathrm{OPh}$. On the other hand, addition of the $\mu$-form seed crystals of $( \pm)-\mathrm{NTMe}_{3}-\mathrm{OPh}$ to the supersaturated EtOH solution of $( \pm)-\mathrm{NTMe}_{3}-\mathrm{OPh}-p \mathrm{~F}$ inhibited the preferential enrichment of $( \pm)-\mathrm{NTMe}_{3}-\mathrm{OPh}-p \mathrm{~F}$ completely, resulting in the deposition of $\mu$-form crystals of $( \pm)-\mathrm{NTMe}_{3}-\mathrm{OPh}-p \mathrm{~F}$.

These results strongly support our proposed mechanism of preferential enrichment including polymorphic transition (Figure 10).

\section{Scope of Preferential Enrichment}

It is quite conceivable that preferential enrichment should be applicable to other chiral organic substances that exist as a fairly ordered racemic mixed crystal, if the three requirements describrd in 
the section 3.6 are satisfied. Furthermore, by carefully choosing the kinetic crystallization conditions or seeding with appropriate crystals, preferential enrichment may be applicable to even certain racemic compounds that satisfy the structural requirements for supramolecular association in solution and in the crystalline state.

Indeed, quite recently we have found that preferential enrichment can be applied to several amino acids, which were believed to be a racemic compound, by carefully choosing kinetic crystallization conditions so as to induce a polymorphic transition [53]. Therefore, we believe that preferntial enrichment will become a potent enatiomeric resolution method of racemic crystals in company with the development of kinetic crystallization technologies.

\section{Conclusions}

The mechanism of preferential enrichment has been interpreted in terms of a symmetry-breaking complexity phenomenon including two major elements; (1) the polymorphic transition of an incipient metastable crystalline form into the more stable one and (2) the dissolution of the excess enantiomer from the transformed crystals into solution, resulting in a considerable enrichment of the excess enantiomer in the mother liquor until a slight enrichment of the opposite enantiomer in the deposited crystals occurs. Therefore, preferential enrichment is not only an excellent chiral separation phenomenon but also a good research model for a complexity phenomenon.

Successful induction of preferential enrichment, based on the concept "epitaxial transition", strongly support the proposed mechanism of preferential enrichment. If the crystal lattices and crystal structures of the two compounds are partly similar to each other, it is possible to control the mode of polymorphic transition mutually by seeding with each other. Accordingly, once one finds a leading racemic sample that can show preferential enrichment, it is possible to induce preferential enrichment for analogous compounds that cannot show preferential enrichment by themselves, on the basis of this epitaxial transition protocol.

Furthermore, preferential enrichment has turned out to be applicable to racemic samples classified as a racemic compound by carefully choosing kinetic crystallization conditions so as to induce a polymorphic transition. Further studies on this subject is currently in progress.

\section{Acknowledgements}

This work was supported by the Grant-in-Aids for Scientific Research from Japan Society for the Promotion of Science, the Mitsubishi Foundation, the Asahi Glass foundation, the Sumitomo foundation, and the Takeda Science Foundation.

\section{References and Notes}

1. Mainzer, K. Symmetry and Complexity: The Sprit and Beauty of Nonlinear Science; World Scientific: Singapore, 2005.

2. Kauffman, S. Investigations; Oxford University Press: Oxford, UK, 2000.

3. Waldrop, M.M. Complexity; Simon \&Schuster: New York, NY, USA, 1992. 
4. Pérez-García, L.; Amabilino, D.B. Spontaneous Resolution Under Supramolecular Control. Chem. Soc. Rev. 2002, 31, 342-356.

5. Ushio, T.; Tamura, R.; Takahashi, H.; Yamamoto, K. Unusual Enantiomeric Resolution Phenomenon Observed upon Recrystallization of a Racemic Compound. Angew. Chem. Int. Ed. Engl. 1996, 35, 2372-2374.

6. Ushio, T.; Tamura, R.; Azuma, N.; Nakamura, K.; Toda, F.; Kobayashi, K. Polymorphism of Compounds Existing as Both Racemic Compounds Crystals and Mixed Crystals of Enantiomers. Mol. Cryst. Liq. Cryst. 1996, 276, 245-252.

7. Tamura, R.; Takahashi, H.; Fujimoto, D.; Ushio, T. Mechanism and Scope of Preferential enrichment, a Symmetry-Breaking Enantiomeric Resolution Phenomenon. Top. Curr. Chem. 2007, 269, 53-82.

8. Tamura, R.; Ushio, T. Preferential Enrichment: A Dynamic Enantiomeric Resolution Phenomenon Caused by Polymorphic Transition During Crystallization. In Enantiomer Separation: Fundamentals and Practical Methods, Toda, F., Ed.; Kluwer: Dordrecht, The Netherlands, 2004; pp. 135-163.

9. Jacques, J.; Collet, A.; Wilen, S.H. Enantiomers, Racemates and Resolutions; Krieger: Malabar, FL, USA, 1994.

10. CRC Handbook of Optical Resolutions via Diastereomeric Salt Formation Kozma, D., Ed.; CRC Press: Boca Raton, FL, USA, 2001.

11. Kinbara, K.; Saigo, K. Chiral Discrimination during Crystallization. Top. Stereochem. 2003, 23, 207-265.

12. Sakai, K.; Sakurai, R.; Nohira, H. New Resolution Technologies Controlled by Chiral Discrimination Mechanisms. Top. Curr. Chem. 2007, 269, 199-231.

13. Kellogg, R.M.; Kaptein, B.; Vries, T.R. Dutch Rresolution of Racemates and the Roles of Solid Solution Formation and Nucleation Inhibition. Top. Curr. Chem. 2007, 269, 159-197.

14. Toda, F. Optical Resolutions by Inclusion Complexation with a Chiral Host Compound. In Enantiomer Separation: Fundamentals and Practical Methods, Toda, F., Ed.; Kluwer: Dordrecht, The Netherlands, 2004; pp. 1- 47.

15. Collet, A.; Brienne, M.-J.; Jacques, J. Optical Resolution by Direct Crystallization of Enantiomer Mixtures. Chem. Rev. 1980, 80, 215-230.

16. Collet, A. Optical Resolution. In Comprehensive Supramolecular Chemistry; Reinhoudt, D.N., Ed.; Pergamon: Oxford, UK, 1996; Vol. 10, pp.113-149.

17. Kinbara, K.; Hashimoto, Y.; Sukegawa, M.; Nohira, H.; Saigo, K. Crystal Structures of the Salts of Chiral Primary Amines with Achiral Carboxylic Acids: Recognition of the CommonlyOccurring Supramolecular Assemblies of hydrogen-Bond Networks and Their Role in the Formation of Conglomerates. J. Am. Chem. Soc. 1996, 118, 3441-3449.

18. Coquerel, G. Preferential Crystallization. Top. Curr. Chem. 2007, 269, 1-51.

19. Eliel, E.; Wilen, S.H.; Mander, L.N. Stereochemistry of Organic Compounds; Wiley: New York, NY, USA, 1994; pp.297-464.

20. Tamura, R.; Takahashi, H.; Hirotsu, K.; Nakajima, Y.; Ushio, T.; Toda, F. Unusual Disordered Crystal Structure of a Racemate Exhibiting a Novel Enantiomeric Resolution: Preferential Enrichment. Angew. Chem. Int. Ed. Engl. 1998, 37, 2876-2878. 
21. Tamura, R.; Fujimoto, D.; Lepp, Z.; Misaki, K.; Miura, H.; Takahashi, H.; Ushio, T.; Nakai, T.; Hirotsu, K. Mechanism of Preferential Enrichment, an Unusual Enantiomeric Resolution Phenomenon Caused by Polymorphic Transition during Crystallization of Mixed Crystals Composed of Two Enantiomers. J. Am. Chem. Soc. 2002, 124, 13139-13153.

22. Fujimoto, D.; Tamura, R.; Lepp, Z.; Takahashi, H.; Ushio, T. Mechanism of a New type of Solvent-Assisted Solid-to-Solid Polymorphic Transition Causing Preferential Enrichment: Prominent Influence of $\mathrm{C}\left(\mathrm{sp}^{2}\right) \mathrm{H}---\mathrm{O}$ Interaction on the Control of a Crystal Structure. Cryst. Growth Des. 2003, 3, 973-979.

23. Tamura R, Mizuta M, Yabunaka S, Fujimoto D, Ariga T, Okuhara S, Ikuma N, Takahashi H, Tsue H. Induction and Inhibition of Preferential Enrichment by Controlling the Mode of the Polymorphic Transition with Seed Crystals. Chem. Eur. J. 2006, 12, 3515-3527.

24. Horiguchi, M.; Okuhara, S.; Shimano, E.; Fujimoto, D.; Takahashi, H.; Tsue, H.; Tamura, R. Control of the Mode of Polymorphic Transition Inducing Preferential Enrichment by Modifying the Molecular Structure or Adding Seed Crystals: Significant Influence of CH/F Hydrogen Bonds. Cryst. Growth Des. 2008, 8, 540-548.

25. Horiguchi, M.; Okuhara, S.; Shimano, E.; Fujimoto, D.; Takahashi, H.; Tsue, H.; Tamura, R. Mechanistic Flexibility of Solvent-Assisted Solid-to-Solid Polymorphic Transition Causing Preferential Enrichment: Significant Contribution of $\pi / \pi$ and $\mathrm{CH} / \pi$ Interactions as Well as Hydrogen Bonds. Cryst. Growth Des. 2007, 7, 1643-1652.

26. Bernstein, J. Polymorphism in Molecular Crystals; Oxford University Press: Oxford, UK, 2002.

27. Polymorphism in Pharmaceutical Solids, Drugs and the Pharmaceutical Sciences; Brittain, H.G., Ed.; Marcel Dekker: New York, NY, USA, 1999; Vol. 95.

28. Bernstein, J.; Davey, R.J.; Henck, J.-O. Concomitant Polymorphs. Angew. Chem. Int. Ed. Engl. 1999, 38, 3440-3461.

29. Dunitz, J.D., Bernstein, J. Disappearing Polymorphs. Acc. Chem. Res. 1995, 28, 193-200.

30. McCrone, W.C. Polymorphism. In Physics and Chemistry of the Organic Solid State; Fox, D.; Labes, M.M.; Weissberger, A., Eds.; Interscience: New York, NY, USA, 1965; Vol. II, pp.726-767.

31. Ostwald, W. Grundriss der Allgemeinen Chemie; W. Engelmann: Leipzig, 1899.

32. Parkinson, G.M.; Thomas, J.M.; Williams, J.O.; Goringe, M.J.; Hobbs, L.W. The Suggested Role of Partial Dislocations in the Single Crystal-Single Crystal Phase Transition of a Cyclo-octane Molecular Cationic Salt. J. Chem. Soc., Perkin 2 1976, 836-838.

33. Tamura, R.; Ushio, T.; Nakamura, K.; Takahashi, H.; Azuma, N; Toda, F. Ideal Enantiomeric Resolution by Recrystallization of a Racemic Compound. Enantiomer 1997, 2, 277-280.

34. Tamura, R., Ushio, T.; Takahashi, H.; Nakamura, K.; Azuma, N.; Toda, F.; Endo, K. Ideal Enantiomeric Resolution of a Racemic Compound (Part 4): Relationship Between Enantiomeric Resolution Phenomenon and Crystal Properties. Chirality 1997, 9, 220-224.

35. Takahashi, H.; Tamura, R.; Ushio, T.; Nakajima, Y.; Hirotsu, K. Ideal Enantiomeric Resolution (Preferential Enrichment) by Recrystallization of a Racemic Compound. V: Relationship Between Preferential Enrichment and Crystal Structures. Chirality 1998, 10, 705-710.

36. Tamura, R.; Takahashi, H.; Hirotsu, K.; Nakajima, Y.; Ushio, T. Preferential Enrichment and Crystal Structure. Mol. Cryst. Liq. Cryst. 2001, 356, 185-194. 
37. Tamura, R.; Takahashi, H.; Ushio, T.; Nakajima, Y.;Hirotsu, K.; Toda, F. Ideal Enantiomeric Resolution (Preferential Enrichment) by Recrystallization of a Racemic Compound (Part 6): Hydrogen Bonding Mode in the Crystal Structure. Enantiomer 1998, 3, 149-157.

38. Tamura, R.; Takahashi, H.; Miura, H.; Lepp, Z.; Nakajima Y.; Hirotsu, K.; Ushio, T. Comparison of Crystal Structures of New Racemic Chiral Compounds Showing and Not Showing the Phenomenon of Preferential Enrichment. Supramol. Chem. 2001, 13, 71-78.

39. Takahashi, H.; Tamura, R.; Lepp, Z.; Kobayashi, K.; Ushio, T. Preferential Enrichment: An Essential Crystal Structure. Enantiomer 2001, 6, 57-66.

40. Takahashi, H.; Tamura, R.; Fujimoto, D.; Lepp, Z.; Kobayashi, K.; Ushio, T. Preferential Enrichment: Full Crystallographic Analysis of the Unusual Phenomenon in the Mixed Crystals' Version. Chirality 2002, 14, 541-547.

41. Takahashi, H.; Tamura, R.; Yabunaka, S.; Ushio, T. Crystal Structure of a New Racemate Showing Preferential Enrichment: Evidence for the Existence as a Racemic Mixed Crystal Composed of the Two Enantiomers. Mendeleev Commun. 2003, 119-121.

42. Miura, H.; Ushio, T.; Nagai, K.; Fujimoto, D.; Lepp, Z.; Takahashi, H.; Tamura, R. Crystallization of a Desired Metastable Polymorph by Pseudoseeding, Crystal Structure Solution from Its Powder X-ray Diffraction Data, and Confirmation of Polymorphic Transition. Cryst. Growth Des. 2003, 3, 959-965.

43. Takahashi, H.; Tamura, R.; Yabunaka, S.; Mizuta, M.; Ikums, N.; Tsue, H.; Ushio, T. Significant Contribution of Phenyl Centroid---I-C( $\left.\mathrm{sp}^{2}\right)$ Coulombic Donor-Acceptor Attraction to the Buildup of a Crystal Structure. Mendeleev Commun. 2004, 239-241.

44. Fujimoto, D.; Takahashi, H.; Ariga, T.; Tamura, R. Preferential Enrichment: Significant Influence of Minor Molecular Modification on the Mode of Polymorphic Transition during Crystallization. Chiraliry, 2006, 18, 188-195.

45. Horiguchi, M.; Yanunaka, S.; Iwama, S.; Shimano, E.; Lepp, Z.; Takahashi, H.; Tsue, H.; Tamura, R. Case Study on the Effects of Molecular Structure on the Mode of Polymorphic Transition Inducing Preferential Enrichment. Eur. J. Org. Chem.2008, 3496-3505.

46. Maruyama, S.; Ooshima, H. Crystallization Behavior of Taltirelin Polymorphs in a Mixture of Water and Methanol. J. Crystal Growth 2000, 212, 239-245.

47. Kitamura, M.; Ueno, S.; Sato, K. Molecular Aspects of the Polymorphic Crystallization of Amino Acids and Lipids. In Crystallization Processes, Ohtaki, H., Ed.; Wiley: Chichester, UK, 1998; pp. 99-129.

48. Kitamura, M. Polymorphism in the Crystallization of L-Glutamic Acid. J. Crystal Growth 1989, 96, 541-546.

49. Dobashi, A.; Saito, N.; Motoyama, Y.; Hara, S. Self-Induced Nonequivalence in the Association of D- and L-Amino Acid Derivatives. J. Am. Chem. Soc. 1986, 108, 307-308.

50. Jursic B.S.; Goldberg, S.I. Enantiomer Discrimination Arising from Solute-Solute Interactions in Partially Resolved Chloroform Solutions of Chiral Carboxamides. J. Org. Chem. 1992, 57, 7172-7174.

51. Harger, M.J.P. Proton Magnetic Resonance Non-equivalence of the Enantiomers of Alkylphenylphosphinic Amides. J. Chem. Soc., Perkin Trans. 2 1977, 1882-1887. 
52. Harger, M.J.P. Chemical Shift Non-equivalence of Enantiomers in the Proton Magnetic Resonance Spectra of Partly Resolved Phosphinothioic Acids. J. Chem. Soc., Perkin Trans. 2 1978, 326-331.

53. Iwama, S.; Horiguchi, M.; Uchida, Y.; Sato, H.; Takahashi, H.; Tsue, H.; Tamura R. Observation of the Preferential Enrichment Phenomenon for Essential Amino Acids. Manuscript submitted for publication. This work was presented in part at the $19^{\text {th }}$ International Conference on the Chemistry of Organic Solid State, Sestri Levante, Italy, 14-19 June 2009.

(C) 2010 by the authors; licensee Molecular Diversity Preservation International, Basel, Switzerland. This article is an open-access article distributed under the terms and conditions of the Creative Commons Attribution license (http://creativecommons.org/licenses/by/3.0/). 